\title{
Encoding and Tracking of Outcome-Specific Expectancy in the Gustatory Cortex of Alert Rats
}

\author{
Matthew P.H. Gardner ${ }^{1,2}$ and Alfredo Fontanini ${ }^{1,2}$ \\ ${ }^{1}$ Department of Neurobiology and Behavior and ${ }^{2}$ Graduate Program in Neuroscience, State University of New York at Stony Brook, Stony Brook, New York \\ 11794
}

In natural conditions, gustatory stimuli are typically expected. Anticipatory and contextual cues provide information that allows animals to predict the availability and the identity of the substance to be ingested. Recording in alert rats trained to self-administer tastants following a go signal revealed that neurons in the primary gustatory cortex (GC) can respond to anticipatory cues. These experiments were optimized to demonstrate that even the most general form of expectation can activate neurons in GC, and did not provide indications on whether cues predicting different tastants could be encoded selectively by GC neurons. Here we recorded single-neuron activity in GC of rats engaged in a task where one auditory cue predicted sucrose, while another predicted quinine. We found that GC neurons respond differentially to the two cues. Cue-selective responses develop in parallel with learning. Comparison between cue and sucrose responses revealed that cues could trigger the activation of anticipatory representations. Additional experiments showed that an expectation of sucrose leads a subset of neurons to produce sucrose-like responses even when the tastant was omitted. Altogether, the data show that primary sensory cortices can encode for cues predicting different outcomes, and that specific expectations result in the activation of anticipatory representations.

Key words: anticipatory cues; coding; expectation; insular cortex; learning; reward

\section{Introduction}

Electrophysiological recordings from alert rodents provide clear evidence that neurons in the gustatory cortex (GC) encode physiochemical (Katz et al., 2001; Jones et al., 2007; Sadacca et al., 2012) and psychological variables associated with taste (Piette et al., 2012; Samuelsen et al., 2012, 2013; Moran and Katz, 2014). Recent evidence has shown that GC can also be recruited in the absence of gustatory stimulation. Approach to a licking spout or to a nose port that allows for self-delivery of tasting solutions is associated with anticipatory changes in firing rates (Stapleton et al., 2007; Yoshida and Katz, 2011). Experiments directly addressing the possibility that these prestimulus modulations could be anticipatory revealed that neurons in GC can robustly respond to auditory cues predicting the general availability of taste (Samuelsen et al., 2012, 2013). Additionally, anticipatory activity has been associated with an improvement in taste coding. Generally, expected tastants are encoded more rapidly and more accurately than stimuli delivered as a surprise (Yoshida and Katz, 2011; Samuelsen et al., 2012).

\footnotetext{
Received May 2, 2014; revised July 10, 2014; accepted Aug. 12, 2014.

Author contributions: M.P.H.G. and A.F. designed research; M.P.H.G. performed research; M.P.H.G. and A.F. analyzed data; M.P.H.G. and A.F. wrote the paper.

This work was supported by National Institute of Deafness and Other Communication Disorders-National Institutes of Health Grant R01-DC010389 and by a Klingenstein Foundation Fellowship (to A.F.). We thank Amy Cheung for her help with histological procedures; and Dr. Arianna Maffei, Dr. Chad Samuelsen, Dr. Ahmad Jezzini, Dr. Luca Mazzucato, Haixin Liu, and Naz Dikecligil for insightful discussions.

Correspondence should be addressed to Matthew P. H. Gardner, Department of Neurobiology and Behavior, Life Science Building, Room 545, SUNY Stony Brook, Stony Brook, NY 11794. E-mail: mphgardner@gmail.com.

DOI:10.1523/JNEUROSCI.1820-14.2014

Copyright $\odot 2014$ the authors $\quad 0270-6474 / 14 / 3413000-18 \$ 15.00 / 0$
}

In nature, however, cues are not just general preparatory signals, they frequently provide information predictive of specific outcomes. While it is clear that neurons in GC can be modulated by the general expectation of taste, it is unknown whether this activity reflects just a general attentional signal (Usher et al., 1999; Roesch et al., 2010) or whether it could represent the expectation of different outcomes (Schoenbaum and Roesch, 2005). Analyses of immediate early gene expression suggested that GC neurons might be activated by the prediction of a specific tastant (Saddoris et al., 2009). This suggestion is compatible with the anatomical organization of GC, which receives inputs from a series of areas known to encode anticipatory cues (Maffei et al., 2012). Yet, to date no evidence is available that single neurons in GC can develop cue-specific responses as the animal learns distinct predictive values.

Here we directly addressed the possibility that GC can respond selectively to cues predicting different outcomes by recording multiple single neurons from rats trained to form specific gustatory expectations. To trigger the specific expectation rats were trained on an auditory go/no-go paradigm, with one cue signaling the availability of sucrose (Suc) and the other signaling the availability of quinine (Q). Sucrose and quinine were chosen based on their difference in sensory quality and palatability, hence maximizing the contrast in predictive value for the two cues. This task, as well as a classical conditioning paradigm, allowed us to unveil cue-specific responses in GC. Cue-specific firing tracked the anticipatory value of cues, as indicated by the study of learning sessions and partial extinction (partial_ext) sessions. A correlation between cue responses and responses to taste was observed. Additional analysis of cue-selective neurons re- 
vealed a group of neurons that produced sucrose-like patterns of activity also in trials in which the delivery of sucrose was unexpectedly omitted.

Altogether, these results provide strong evidence that single neurons in GC can be recruited differentially by cues predicting different tastants and emphasize the importance of expectation in activating anticipatory representations.

\section{Materials and Methods}

\section{Experimental subjects}

All experimental procedures were approved by the Institutional Animal Care and Use Committee of Stony Brook University, and complied with university, state, and federal regulations on the care and use of laboratory animals. The 31 female Long-Evans rats (275-350 g; Charles River) used for this study were individually housed and maintained on a $12 \mathrm{~h} \mathrm{light/}$ dark cycle with ad libitum access to chow and water, unless otherwise specified. Facilities were temperature and humidity controlled.

\section{Surgery}

Rats were anesthetized using a ketamine/xylazine/acepromazine mixture $(100,5.2$, and $1 \mathrm{mg} / \mathrm{kg}$, respectively) injected intraperitoneally with supplemental doses ( $30 \%$ of induction dose) that were administered as needed. Temperature was maintained at $37^{\circ} \mathrm{C}$ throughout the surgery. Animals were placed on a stereotaxic device, the skull was exposed, and holes were drilled above GC $(+1.4$ anteroposterior and \pm 5.0 mediolateral relative to bregma; -4.0 dorsoventral from dura) for electrode implantation, and in seven other positions to secure $0-80$ head screws. The electrode consisted of drivable bundles of 16 individual nichrome wires with formvar coating (Samuelsen et al., 2013). Movable bundles allowed us to record multiple ensembles in the same animal. Following insertion of the electrode bundles, intraoral cannulae (IOCs; polyethylene tubing; outer diameter, $1.70 \mathrm{~mm}$ ) were bilaterally inserted into the mouth for direct delivery of tastes (Phillips and Norgren, 1970; Fontanini and Katz, 2006). The IOCs, electrode bundles, and a bolt (for the purpose of head restraint) were cemented to the skull and head screws using dental acrylic. Animals recovered for a minimum of 7 days and were required to reach $85 \%$ of their original body weight before training began. In addition to undergoing the surgical procedures outlined above, rats trained in the classical conditioning paradigm were implanted with insulated stainless steel wires ( 0.003 inches bare, 0.0055 inches coated) into the anterior digastric muscle to record electromyographic (EMG) activity (Travers and Norgren, 1986). Two wires with a $1 \mathrm{~mm}$ uninsulated tip were implanted a few millimeters apart into the muscle and fed up to a connector attached to the head cap with additional dental acrylic.

\section{Behavioral paradigms}

Following recovery, rats were put on a mild water restriction regimen (45 min of free access to water each day) 1 week before the beginning of training. Animals were progressively habituated to receive fluids via an IOC, to calmly sit while restrained, and to freely press the lever for water $(40 \mu \mathrm{l})$. Session durations and intertrial intervals (ITIs) were progressively increased to $60-90 \mathrm{~min}$ and to $35 \pm 5 \mathrm{~s}$, respectively, over the first $10-15 \mathrm{~d}$ of training. Animals were then trained that the ITI terminated with the onset of a 3 s tone $(4.5 \mathrm{kHz}, 80 \mathrm{~dB})$ and were required to press the lever during the $3 \mathrm{~s}$ cue period to receive water. Pressing during the cue period terminated the tone and led to water delivery. Both lever press with water delivery and failure to press within the cue period initiated the beginning of a new trial. Early presses or presses within the ITI period were discouraged by increasing the ITI by $2 \mathrm{~s}$. Sessions typically lasted $\sim 60-90 \mathrm{~min}$ and were terminated at signs of disengagement (Fontanini and Katz, 2005). Once rats achieved minimal pressing during the ITI $(0-2$ presses/trial), a response percentage of $>90 \%$, and an ITI of $35 \pm$ $5 \mathrm{~s}$; animals began training on the two-tone, go/no-go paradigm. Between 4 and 6 weeks of training and shaping were normally required to reach this stage.

Two-tone, go/no-go paradigm. In this paradigm, rats learned to lever press for a rewarding stimuli, Suc $(0.1 \mathrm{M})$, and to withhold from pressing for an aversive stimulus, Q (5-10 mM during training, $10 \mathrm{~mm}$ following training). High concentrations of $\mathrm{Q}$ were required to achieve optimal no-go behavior. The switch in the concentration of Q from 5 to $10 \mathrm{~mm}$ was motivated by the observation that over the first days of go/no-go training, some rats tended to habituate to $5 \mathrm{~mm}$ and began to perform less consistently in no-go trials. To ensure consistent and robust performance, rats were switched to $10 \mathrm{~mm}$ in the last stages of training before experimental sessions (well trained go/no-go sessions). Each taste was paired with one of two pure tones (typically, 2 and $10 \mathrm{kHz}$ counterbalanced across animals; $80 \mathrm{~dB}$ ), so that one tone indicated the availability of Suc solution and the other of Q solution. Auditory cues began with the termination of the ITI ( $35 \pm 5 \mathrm{~s}$ ) and lasted for a maximum of $3 \mathrm{~s}$. Lever presses within the cue period immediately resulted in the delivery of the cue-associated tastes and the termination of the cue. Both taste deliveries and failure to press within the cue period initiated the beginning of a new trial. Trial types, Suc or Q, were chosen pseudorandomly with a 1:1 ratio. Animals were trained on this task until they showed learning of the two cues (i.e., $80 \%$ correct performance). Correct performance was defined as lever pressing to the sucrose-associated cue (Suc_cue) and withholding of pressing to the quinine-associated cue (Q_cue). Correct performance was measured using a 10 trial moving window. Animals typically learned the task within one to five sessions, each lasting between $\sim 60$ and $90 \mathrm{~min}$. Sessions were terminated when the animal stopped pressing over 10 consecutive trials and showed signs of disengagement. The ability to remain task oriented appeared to be longer than in an uncued selfadministration task previously published (Fontanini and Katz, 2005, 2006). The difference is likely related to the use of salient cues and highly rewarding Suc in the present task. Testing sessions involved the addition of a water rinse $(50 \mu \mathrm{l})$ delivered $10 \mathrm{~s}$ following taste deliveries $(40 \mu \mathrm{l})$.

Unexpected sucrose sessions. Unexpected Suc sessions were go/no-go sessions in which Suc was unexpectedly delivered through the IOC at random trials and intervals during the ITI period. These deliveries were identical to those elicited by lever press, differing only by the lack of cue and lever press. Deliveries were triggered by the experimenter to prevent delivery overlap with aberrant lever-press movements or grooming behaviors. Suc deliveries were followed by a water rinse $(50 \mu \mathrm{l})$ delivered $10 \mathrm{~s}$ later. No taste, passive or self-delivered, occurred within $15 \mathrm{~s}$ of the prior rinse. The ITI during trials with passive deliveries was increased by $25 \mathrm{~s}$, including the $10 \mathrm{~s}$ rinse delay. Typically, 8-12 deliveries of Suc were delivered during a session. Unexpected deliveries were performed only after the animal had reached the $80 \%$ correct performance level.

Omission sessions. Omission sessions were go/no-go session in which $20 \%$ of the Suc_cue trials did not lead to Suc delivery following the lever press. Instead, in these trials a lever press during the cue period stopped the cue, no tastant was delivered, and a rinse was delivered after $10 \mathrm{~s}$. The rinse was delivered so that omission trials differed only in the lack of Suc delivery. These trials were given only after the animal had reached the $80 \%$ correct percentage criterion.

Extinction sessions. Partial extinction sessions were performed to determine whether cue-responsive neurons tracked the cue-outcome association. Partial extinction was preferred over reversal due to its feasibility within a single session; in preliminary experiments, reversal required long, multiday training to be achieved. Extinction sessions began as regular go/no-go sessions. Once animals had completed 30 trials after reaching the $80 \%$ correct criterion, extinction of the cue-taste pairing began. During extinction trials, a lever press occurring in the cue period ended the current trial with no taste and rinse delivery. The percentage of pressing to Suc_cue was assessed over 10 prior trials; when the animal pressed for $\leq 40 \%$ of the Suc_cue trials, the animal was considered to have partially extinguished the conditioned response behavior. The session terminated when animals withheld from pressing in at least 15 Suc trials.

Classical conditioning sessions. As a control for the effects of lever pressing, two rats were trained to associate auditory tones with tastants (Suc and Q) in a pavlovian task. Rats underwent the same water restriction regimen and habituation to IOC fluid deliveries as animals in the operant task. Animals were then trained to calmly receive water $(40 \mu \mathrm{l})$ while head restrained. Session duration and ITI were progressively increased to $1 \mathrm{~h}$ and to $35 \pm 20 \mathrm{~s}$, respectively, over the first $10-15 \mathrm{~d}$ of training. Animals were then habituated for one session to receive $40 \mu \mathrm{l}$ of Suc $(0.1$ $\mathrm{M})$ and $\mathrm{Q}(1 \mathrm{~mm})$, followed by a $50 \mu \mathrm{l}$ rinse (10 s after). Following this day 
of taste habituation, rats were trained for $10 \mathrm{~d}$ to associate one auditory cue with Suc and another with Q deliveries. Trials consisted of a $35 \pm 20 \mathrm{~s}$ ITI followed by a $2 \mathrm{~s}$ tone with taste deliveries occurring $1 \mathrm{~s}$ following the offset of the tone. Auditory cues (rat 1: Suc_cue, $11.0 \mathrm{kHz}$ pure tone; Q_cue, 2.5-9.0 kHz bandpass-filtered Gaussian noise; rat 2: Suc_cue, 0.5 $\mathrm{kHz}$ pure tone; Q_cue, $2.5 \mathrm{kHz}$ amplitude-modulated sawtooth at 100 $\mathrm{Hz}$; all tones were delivered at $80 \mathrm{~dB}$ ) were kept consistent throughout training and testing. Learning was assessed by monitoring the emergence of differential conditioned responses to the two cues. Conditioned responses consisted of mouth movements, which were assessed and quantified by EMG recordings of jaw-opening muscles. Only recordings from sessions in which learning was demonstrated were used for analysis.

Taste deliveries. Tastes were delivered through the IOCs in $40 \mu \mathrm{l}$ aliquots (delivery time of $40 \mathrm{~ms}$ ) by a pressurized taste delivery system (20 psi) operating with computer-controlled solenoid valves. A $50 \mu$ l rinse of water followed the taste $10 \mathrm{~s}$ after to wash the tongue and palate. The Suc and $Q$ concentrations used during testing were $0.1 \mathrm{M}$ and $10 \mathrm{~mm}$, respectively. Although several sessions were analyzed in which Q was delivered at $5 \mathrm{~mm}$ [i.e., initial go/no-go training sessions; sessions using $5 \mathrm{~mm}$ were balanced across conditions on the first day on which animals were trained on the go/no-go task, yet did not reach the criterion (no_learn) and those on the first day on which animals reached the criterion (first_learn)], only cue responses were analyzed within these sessions.

\section{Electrophysiological and video recordings}

During each recording session activity was recorded from movable bundles positioned in GC (either unilaterally or bilaterally). Upon the termination of a set of different sessions [which could involve the following: well trained go/no-go, no_learn, first_learn, partial_ext, "unexpected sucrose," and omission sessions] electrodes were advanced a minimum of $80 \mu \mathrm{m}$ to record new ensembles. The average number of units recorded per rat in the go/no-go experiment was $33.3 \pm 6.9(n=29)$. The average number of units recorded per rat in the classical conditioning experiment was $54.5(n=2)$. Neural signals were simultaneously amplified (gain of $4000-16,000)$ then separately bandpass filtered [at $300-8000 \mathrm{~Hz}$ for single units and $0.7-300 \mathrm{~Hz}$ for local field potentials (LFP)], digitized, and recorded (sampling rate: $40 \mathrm{kHz}$ for single-unit waveforms, $1 \mathrm{kHz}$ for LFP; Multichannel Acquisition Processor, Plexon). Unique single-unit waveforms with at least a 3:1 signal-to-noise ratio were isolated on-line using voltage threshold detection and a template algorithm. Units were further sorted off-line using cluster-cutting techniques and examination of interspike interval plots (Offline Sorter, Plexon). Orofacial reactions to tastants and tones were video recorded (30 frames/s) with videos synchronized to the electrophysiological recordings (Cineplex, Plexon).

\section{Analysis of electrophysiological data}

Spike sorting and data analysis were performed using Offline Sorter (Plexon), Neuroexplorer (Nex Technologies), and custom-written scripts in MATLAB (MathWorks). To compare neural activity with behavioral events, time stamps of single-unit spiking activity were aligned with stimuli of interest in the task. Perievent rasters of individual units were used to generate peristimulus time histograms (PSTHs). Unless otherwise stated, nonparametric tests were used for statistical comparisons due to their robustness and to avoid the assumption that data were normally distributed. Indeed, normality tests often revealed that data were not normally distributed (at least for the bin widths used).

Population PSTHs and auROC normalization. For neural population analyses and plots, PSTHs were normalized using the nonparametric, area under the receiver operating characteristic curve (auROC) method (Cohen et al., 2012; Chubykin et al., 2013). This method normalizes stimulus-related activity to baseline activity on a $0-1$ scale in which 0.5 represents a median equivalence to baseline firing rate. auROC normalization results in values ranging from 0 to 1 , with a value of 0.5 corresponding to baseline, and values $>0.5$ or $<0.5$, respectively, being excitatory or inhibitory responses. auROC values are based on the probability that values within a given bin are higher or lower than baseline activity. A value of 1 indicates that all values within the bin of interest are higher than all values within the baseline window, whereas a value of 0 indicates all values within the bin are less than all values within the baseline window. Population PSTHs (popPSTHs) were created by averaging the auROC normalized firing rate of all neurons within a subpopulation with error bars representing the mean SE at each bin. The difference in firing rates evoked by Suc_cue and Q_cue (i.e., $\triangle$ PSTH) was determined by averaging the absolute difference in cue-evoked activity, across the cues, for each cue-responsive unit and subtracting baseline difference.

Cue responsiveness. PSTHs for single units were aligned to the onset of either the Suc_cue or Q_cue. Cue-evoked responses were assessed for 250 $\mathrm{ms}$ following the onset of the cue over bins of $50 \mathrm{~ms}$. The choice of a 250 ms window for analysis of cue responses was based on a quantification of the latency of lever pressing. Since rats were free to press at any time after the cue (within a $3 \mathrm{~s}$ window), reaction times varied from trial to trial. The average latency of all lever presses was $696 \pm 4 \mathrm{~ms}$, the average latency of the fastest press for each session was $330 \pm 8 \mathrm{~ms}$. Using a 250 ms window resulted in the removal of only $1.6 \%$ ( $n=188$ of 11,454 total trials) of the trials (which had lever pressing earlier than $250 \mathrm{~ms}$ ). Analysis of the distribution of lever presses revealed that use of larger window widths would have led to removal of a substantially larger number of trials (i.e., $5.9 \%$ for $300 \mathrm{~ms}, 12.2 \%$ for $350 \mathrm{~ms}$, and $38.5 \%$ for $500 \mathrm{~ms}$ ). In addition, extending the window to longer intervals would have led to the systematic inclusion of mouth movements (whose average onset in the case of Suc was $348.6 \pm 19.1 \mathrm{~ms}$ ), which would have represented a potential confound. Finally, a 250-ms-wide window was consistent with prior investigations of cue responses in a self-administration task published by our group (Samuelsen et al., 2012). Each bin following cue onset was compared with $1 \mathrm{~s}$ of baseline using an unbalanced Wilcoxon rank-sum test $(p<0.05)$ with correction for familywise error accomplished by requiring either one bin to pass with a Sidak $\alpha$ correction or two consecutive bins to pass with $p<0.05$. Units with a significant increase in activity following the cue were deemed excitatory cue responsive, whereas neurons with a significant decrease in activity were deemed inhibitory cue responsive. Only correct postcriterion trials were used in this analysis, and a minimum of six trials were required for inclusion of a unit. Cue-responsive units were split into the following four groups based on their responsiveness to the two cues: exclusively Suc_cue responsive, exclusively Q_cue responsive, responsive to both but significantly different, responsive to both and not significantly different. Significant difference between cue responses was assessed using a Wilcoxon rank-sum test $(p<0.05)$ comparing bin-to-bin activity of the two cues. Correction for familywise error was accomplished by requiring either one bin to pass with a Sidak $\alpha$ correction or two consecutive bins to pass with $p<0.05$. Peak responsiveness for each neuron was determined as the maximum evoked normalized firing rate (auROC) across the five $50 \mathrm{~ms}$ bins.

Comparison of lever press-evoked activity. To determine whether cueevoked activity was due to the motor act of pressing the lever, responses to lever presses that occurred after the cue onset were compared with responses to lever presses that occurred during the ITI. Rats largely pressed only once following the cue (Fig. $1 C$ ). To match this condition, only ITI lever presses that occurred in isolation (i.e., that were not preceded or followed by a press for a $1 \mathrm{~s}$ interval) were considered for this analysis. A minimum of eight lever presses in each condition was required for the analysis to achieve statistical power. One hundred thirtyseven of the 158 Suc_cue-responsive neurons came from sessions that met these criteria. The normalized firing rate of the $50 \mathrm{~ms}$ before lever presses was compared using a Wilcoxon signed-rank test.

Learning session analyses. Analysis of learning sessions was split into the following two conditions: no_learn sessions (12 animals, 12 sessions); and first_learn sessions (18 rats, 18 sessions). Six of the 29 rats either took $>3 \mathrm{~d}$ to learn the task or were removed from the analysis due to experimenter intervention during the task or lack of recordings. In 5 of the 23 sessions, animals demonstrated a biased behavior toward one of the cues in the first quarter of the session and were removed from the analysis (>60\% correct responding). This was done to avoid inflating any biases toward either of the cues in the analysis and provide a conservative estimate of cue selectivity.

Analysis of cue responses was the same for both conditions. Cue responsiveness was computed using all trials in the session; only those trials 
A GC Electrode Locations

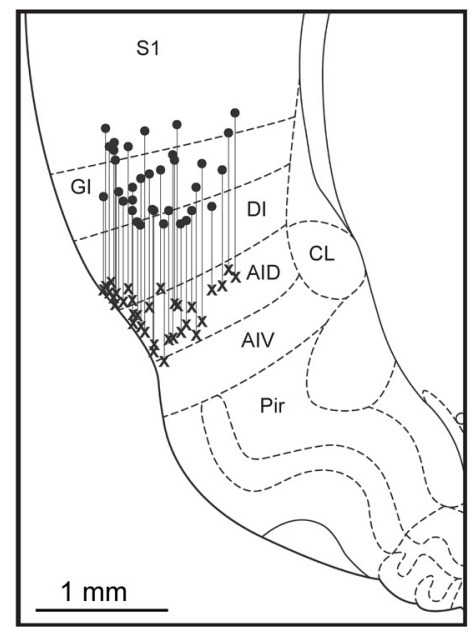

Bregma: 1.44

C

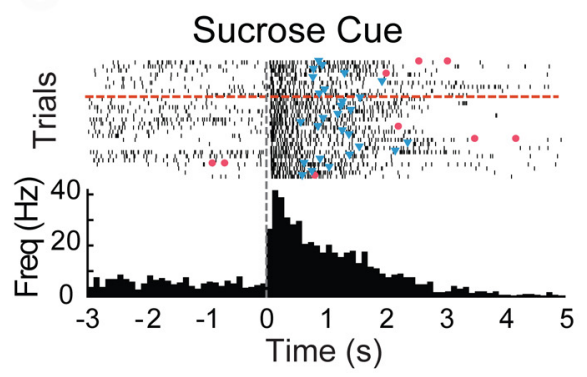

Quinine Cue
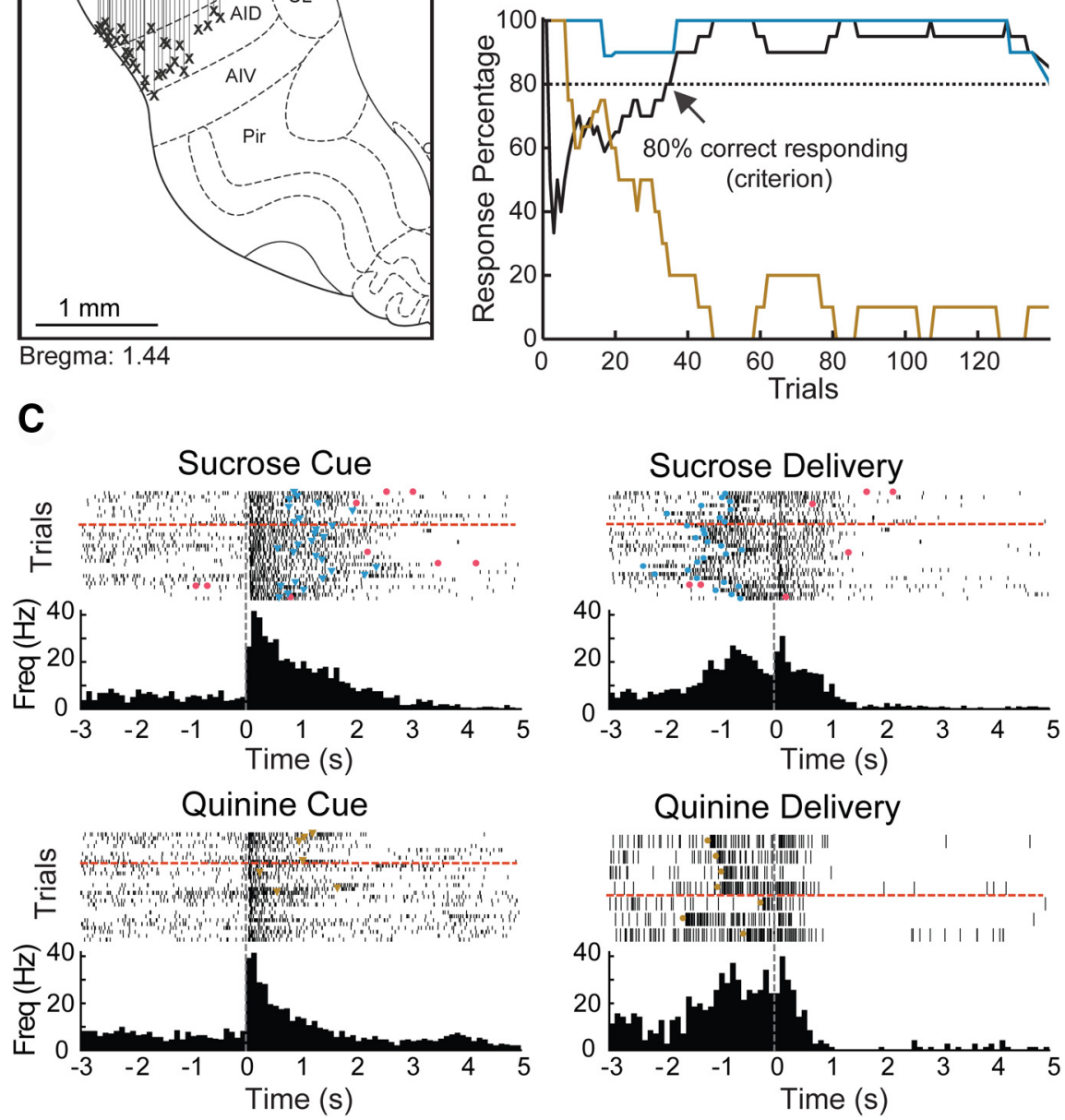

Figure 1. Experimental design. $A$, Schematic representing the reconstructed positioning of recording electrodes in $G$ C. Each line represents the positioning of a movable bundle along the dorsoventral axis. Circles, Starting point of recordings; crosses, final recording site. Positioning was reconstructed according to initial stereotaxic coordinates, electrode advancement after a group of sessions, and histological identification of the terminal recording site. S1, Somatosensory cortex; GI, granular insular cortex; DI, disgranular insular cortex; AID, dorsal agranular insular cortex; AIV, ventral agranular insular cortex; CL, claustrum; Pir, piriform cortex. B, Behavioral paradigm: auditory go/no-go task. Top, Design of each trial. Bottom, A representative session. $x$-Axis, trials; $y$-axis, percentage of cues followed by a lever press; cyan line, Suc trials; gold line, $Q$ trials; black line, performance averaged across cues; dotted line, level of criterion performance. Performance was computed using a moving window of 10 trials and a step of 1 trial. C, Raster plots and PSTHs for a representative cue-responsive neuron during a complete session. Left panels, Raster plots and PSTHs in response to Suc_cue (top) and Q_cue (bottom). Vertical line at time 0 indicates the onset of the auditory cue. Right panels, Raster plots and PSTHs in response to self-delivery of Suc (top) and Q (bottom). Vertical line at time 0 indicates the time of lever pressing, self-delivery, and the simultaneous offset of the cue. Cyan triangle, Time of Suc self-delivery; gold triangle, time of $Q$ self-delivery; cyan circle, time of Suc_cue onset; gold circle, time of Q_cue onset; red circle, ITI lever presses (i.e., extra presses not leading to self-delivery); dashed horizontal line, trial at which criterion is achieved. In the raster plots, the top rows represent earlier trials.

with lever presses earlier than $250 \mathrm{~ms}$ were removed. The distributions of cue-responsive neurons in different sessions were compared using a $2 \times$ 4 Fisher's exact test. The population $\triangle$ PSTHs between cue responses were computed across all cue-responsive neurons for the entire session for each condition. The significance of $\triangle$ PSTHs and the differences in $\Delta$ PSTHs for the various sessions were assessed using a Wilcoxon signed- rank test. To analyze the within-session time course of learning and cue responsiveness, sessions were split into four epochs, each consisting of $25 \%$ of the trials. The correct response percentage was computed for each quarter of individual sessions and averaged across all sessions for each of the conditions. The number of cue-responsive neurons that differentiated between the cues was assessed in each of these quarters. The significant differences between cue responses were assessed using a Wilcoxon rank-sum test $(p<0.05)$ comparing the binto-bin activity of the two cues. Correction for familywise error was accomplished by requiring either one bin to pass with a Sidak $\alpha$ correction or two consecutive bins to pass with $p<0.05$. A one-sided $\mathrm{McNemar}$ test was used to determine whether the number of differentiating neurons increased between the first and last quarter of the sessions. A Fisher's exact test was used to compare the number of differentiating neurons in no_learn sessions to first_learn sessions for each quarter.

Partial extinction. Within extinction sessions, auROC-normalized activity of Suc_cueresponsive neurons was compared before and after partial extinction. Pre-extinction activity was taken from correct postcriterion Suc trials before the beginning of the extinction period, whereas partial extinction activity was taken from trials in which the rat withheld from pressing during the partial extinction period ( $\leq 40 \%$ lever pressing to Suc_cue). Since activity for each unit is matched, a Wilcoxon signed-rank test $(p<0.05)$ was used to assess whether cue-evoked activity significantly decreased following partial extinction in (1) all cue-responsive neurons and (2) Suc_cueselective neurons. To include both excitatoryand inhibitory-responsive neurons, cue response magnitudes were determined by subtracting the baseline activity and taking the absolute value. Partial extinction sessions were compared with time-matched basic go/no-go sessions to check whether a time effect could lead to a decrease in cue-evoked activity. Early postcriterion Suc trials (1-10) were compared with late postcriterion Suc trials (31-40) using a Wilcoxon signed-rank test.

Comparison of sucrose taste responses. Suc responses were compared across groups of cueresponsive neurons (Suc_cue selective and Q_cue selective) using a two-way ANOVA over $2 \mathrm{~s}$ following the taste delivery in $250 \mathrm{~ms}$ bins. The cumulative distribution function was computed on the bin showing the largest difference in the post hoc analysis. To assess whether the strength of cue responses was related to taste responses, neurons were first grouped based on the following quartiles of cue strength: (1) all; (2) top 75\%; (3) top 50\%; and (4) top $25 \%$. One-sided Wilcoxon rank-sum tests were then performed to determine whether the magnitude of the Suc taste response was larger in the Suc_cue neurons than the Q_cue neurons.

To compare whether Suc_cue neurons had a similar taste response to their cue response, activity was analyzed following taste delivery. The first $250 \mathrm{~ms}$ following taste onset, the same time window as for the cue, was compared with $1 \mathrm{~s}$ of baseline ( $5 \mathrm{~s}$ before taste delivery to avoid cue 
contamination) using an unbalanced Wilcoxon rank-sum test $(p<$ 0.05). The $1 \mathrm{~s}$ baseline chosen for the analysis of taste responses was adjacent (yet not overlapping) to the $1 \mathrm{~s}$ baseline used for the cue. Taste responses were considered excitatory or inhibitory based on the sign of the significance test. This same analysis was used for determining neural responsiveness to unexpected and omitted Suc deliveries. Neurons were defined as "matching" if their cue response type matched their taste response type. Distributions of response types (excitatory, inhibitory, and nonsignificant) were compared across cue types using $\chi^{2}$ tests.

Correlation between responses. Linear regression analysis was used to determine the coefficient of determination $\left(R^{2}\right)$ between normalized evoked responses in neurons under different conditions (unexpected vs expected, omitted vs expected, ITI presses vs cued lever presses). The significance of the correlation was determined using a bootstrap (number of bootstraps, 10,000) of shuffled responses across neurons (Narayanan and Laubach, 2009). The bootstrap procedure was used to create an empirical probability distribution, from which a $95 \%$ confidence interval for the correlation between responses was estimated. Statistical significance of the results was maintained also using a lower number of bootstraps $(n=1000)$.

Mouth movements and orofacial behaviors. Mouth movements were assessed by a custom-built automated frame-by-frame video analysis of the orofacial region (Samuelsen et al., 2012, 2013). Videos (30 frames/s, AVI format) of experimental sessions were imported into MATLAB, and time stamps of frames were acquired from Cineplex software (Plexon). The rat orofacial region was isolated by cropping the image, and the absolute difference in pixel intensity across consecutive frames was computed. These differences were then averaged over the entire cropped image, providing a single-value estimate ( $\Delta$ pixel intensity/frame) of mouth movement over a $33 \mathrm{~ms}$ period. Video segments around the auditory cues, $1 \mathrm{~s}$ before and $2 \mathrm{~s}$ after, were used for analysis. Only trials in which the mouth was not already in motion and had a clear, unobstructed view of the orofacial region were included. The time course of the change in pixel intensity was averaged for each trial type to analyze Q_cue and Suc_cue responses separately. This analysis was confirmed with blind visual inspection. Video analysis of mouth movements was performed on $37.7 \%$ of the total number of well trained go/no-go sessions (i.e., on 104 of 276 sessions). Such an analysis was not performed on the entire set of well trained go/no-go sessions due to the computationally intensive nature of the image analysis software and the time-intensive nature of blind manual validation. The onsets of mouth movement and cue responses were computed for the same trials and sessions using a cumulative sum-based method (Samuelsen et al., 2012, 2013). For appropriate comparison, single-unit PSTHs were analyzed using $33 \mathrm{~ms}$ bins, a bin size equivalent to the frame rate. The cumulative sums of both the neural firing rate and the average change in pixel intensity were computed over a $3 \mathrm{~s}$ window, $1 \mathrm{~s}$ before cue onset and $2 \mathrm{~s}$ following the cue. Then a bootstrap (number of bootstraps, 10,000 and 1000) of the $1 \mathrm{~s}$ baseline period was computed, and the first bin that significantly differed from this distribution $(p<0.05)$ was defined as the latency of response or mouth movement. A total of $98.7 \%$ of the neurons in this analysis ( $n=$ 76 of 77) had a faster cue onset than mouth movement within the same trials. A Wilcoxon signed-rank test was used to assess whether cue onsets preceded mouth movement onsets. The magnitude of the cue-evoked mouth movements as a percentage of the Suc-evoked mouth movements was determined by taking the average absolute difference in pixel intensity over the $250 \mathrm{~ms}$ following the cue and dividing by the average absolute difference in pixel intensity over the $250 \mathrm{~ms}$ following the delivery of Suc.

\section{Electromyographic recordings and analysis}

EMG activity was recorded from the anterior digastric muscle during the classical conditioning paradigm to quantitatively assess orofacial reactive behaviors (Travers and Norgren, 1986). Differential electric potentials were amplified (gain, 10,000 Hz), bandpass filtered $(300-1000 \mathrm{~Hz}$ ), and subsequently recorded and digitized with a sampling rate of $4 \mathrm{kHz}$ (Multichannel Acquisition Processor, Plexon). For analysis of mouth movements, EMG signals were imported into MATLAB, rectified, and smoothed to identify single motor movements defined by the crossing of a threshold ( $4 \sigma$ of the EMG signal). Bouts of motor movements (series of single-motor events) were identified using custom-written MATLAB scripts and empirically verified. To verify learning, the duration of bouts evoked by the cues was compared across the Suc_cue and Q_cue for each animal using a Wilcoxon rank-sum test ( $p<0.01$ for both rats), with the Suc_cue evoking increased motor bouts relative to the Q_cue.

At the end of experimental sessions, rats were anesthetized (using the mix mentioned above), and DC current ( $7 \mu \mathrm{A}, 7 \mathrm{~s}$ ) was applied to several electrode wires to mark recording sites. Subjects were then intracardially perfused with saline followed by $10 \%$ formalin. Brains were sectioned into $80 \mu \mathrm{m}$ coronal slices, and standard histological procedures (staining with Prussian blue followed by cresyl violet) were performed to track electrode locations (Samuelsen et al., 2013).

\section{Results}

Firing activity was recorded from ensembles of single neurons in the GC of 31 rats using chronically implanted bundles of electrodes. Figure $1 A$ shows the positioning of electrodes and the dorsoventral range of the recordings. Rats were trained to successfully perform an auditory go/no-go task. Ensembles of neurons were recorded in multiple variants of the go/no-go task with the following breakdown for types of sessions: well trained go/ no-go test sessions (29 rats, 276 sessions, 965 neurons; Fig. 1B); first go/no-go training sessions showing no signs of learning (12 rats, 12 sessions, 95 neurons; see Fig. 5A, left); initial go/no-go training sessions showing learning (18 rats, 18 sessions, 108 neurons; see Fig. 5B, left); partial extinction sessions (13 rats, 27 sessions, 127 neurons; see Fig. $6 A$ ); unexpected sucrose sessions (16 rats, 51 sessions 302 neurons; see Fig. 9); and omission sessions (15 rats, 75 sessions, 419 neurons; see Fig. 10). Two additional rats ( 2 rats, 27 sessions, 109 neurons) were used for classical conditioning sessions.

\section{GC neurons can be selectively activated by cues anticipating either rewarding or aversive outcomes}

Head-restrained rats were trained to perform an auditory go/ no-go task (Fig. 1B, top). Upon successful learning of the task, water-restricted rats typically behaved as in the representative test session featured in Figure $1 B$. In the initial portion of the test session, fully trained rats indistinguishably pressed the lever to both cues, likely a motivational effect. After an average of $35.87 \pm$ 1.26 trials, rats began to respond differently to the two cues; they continued to press following the Suc_cue and reduced pressing to the Q_cue. Neural responses to cues were analyzed after rats reached and maintained $80 \%$ correct performance (Gutierrez et al., 2010). Cues were found to significantly affect firing activity in $29.7 \%$ of recorded GC neurons (287 of 965 neurons). Figure $1 C$ shows an example of the firing activity of a neuron and the lever pressing by a rat recorded throughout a session; each raster and PSTH shows activity from $3 \mathrm{~s}$ before to $5 \mathrm{~s}$ after the event (cue presentations or taste self-deliveries). Lever pressing was largely limited to one press after the cue and occasional presses in the ITI.

Observation of individual neurons revealed that responses to cues could have different degrees of selectivity. Figure $2 A$ shows raster plots and PSTHs in response to Suc_cue and Q_cue for the following four representative cue-responsive neurons: one firing selectively to Suc_cue (cyan); one firing selectively to Q_cue (gold); one firing to both cues, but more strongly to Q_cue (magenta); and, finally, one firing equally to the two cues (black). Superimposed on the raster plots are the time stamps reflecting the time of self-delivery (triangles). Superimposed on the PSTHs are the traces showing the time course of mouth movements detected via an automated frame-by-frame analysis using image analysis software (dashed red lines). To analyze the overall re- 


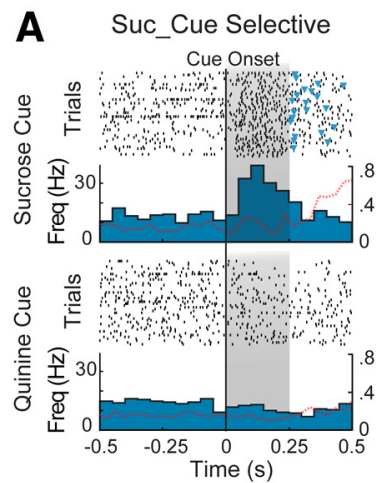

B

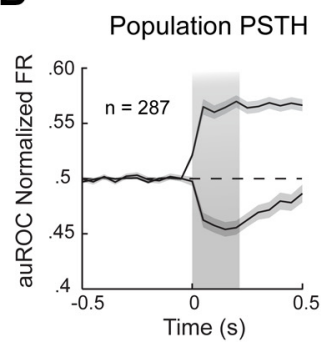

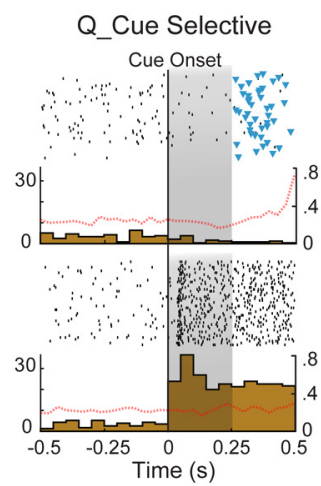

C

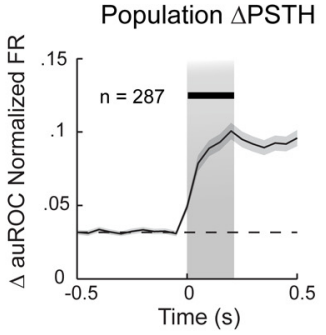

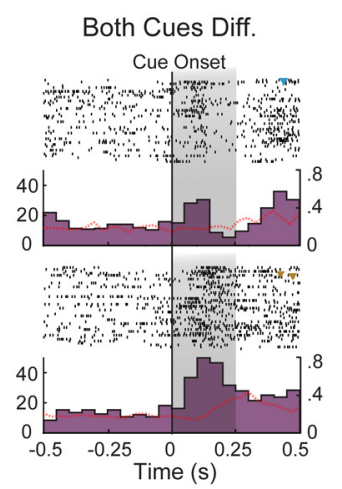

D

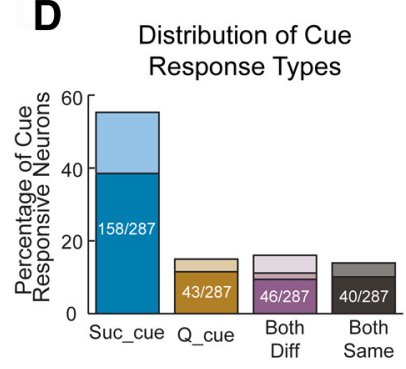

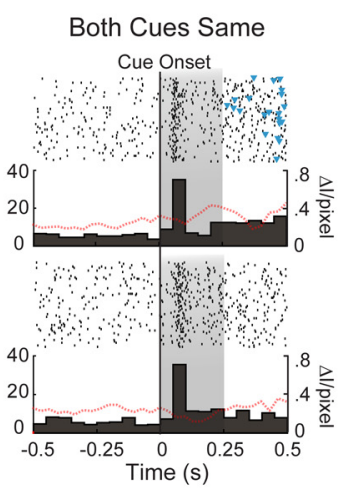

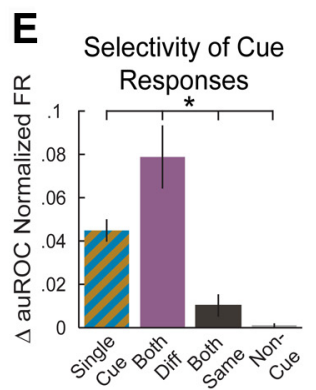

Figure 2. Neurons in the GC of well trained rats respond to specific cues. $A$, Representative raster plots and PSTHs of a Suc_cue-selective neuron (cyan); $\mathrm{Q}$ _cue-selective neuron (gold); a neuron responding to both cues, but with stronger responses to $Q \_$cue (magenta); and a neuron responding equally to both cues (black). Top two panels, Responses to Suc_cue; bottom two panels, responses to Q_cue; triangles in the rasters, time at which the tastant was self-administered (cyan, Suc; gold, Q); red dashed line superimposed on the PSTHs, time course of mouth movements; $x$-axis, time (vertical line 0 indicates the onset of the cue); $y$-axis for rasters, trials; $y$-axis for PSTHs, firing frequency; $y$-axis for mouth movements, difference of intensity for adjacent pixels. $\boldsymbol{B}$, Population PSTH of excitatory and inhibitory responses averaged across cues. C, Absolute difference between responses to Suc_cue and Q_cue averaged across all cue-responsive neurons. The thick line above the trace represents a significant difference from baseline. For $\boldsymbol{A}-\boldsymbol{C}: x$-axis, time ( 0 is the onset of the cue); $y$-axis, auROC; shading around traces, SEM. Shaded vertical rectangles show the time window in which cue responsiveness was considered. $\boldsymbol{D}$, Distribution of cue-responsive neurons as follows: Suc_cue-selective (cyan); $Q$ _cue-selective (gold); differentially responding to both cues (magenta); and equally responding to both cues (black). Dark shades, Excitatory responses; light shades, inhibitory responses; intermediate shade of magenta, neurons with excitatory and inhibitory responses depending on the cue. $\boldsymbol{E}$, Quantification of selectivity on the basis of $\triangle$ PSTH. Cyan/gold stripes, Suc_cue- and Q_cue-selective neurons; magenta, neurons differentially responding to both cues; black, neurons equally responding to both cues; gray bar, neurons not responsive to cue. The asterisk indicates significant differences across all combinations.

sponsiveness to cues in the population of neurons, we computed across-neuron averages of excitatory and inhibitory responses (i.e., popPSTH) combined across cues (Fig. 2B). A total of 70.4\% of the cue-responsive neurons (202 of 287 neurons) was significantly excited, and $25.4 \%$ ( 73 of 287 neurons) was inhibited by at least one of the tones; $4.2 \%$ of neurons ( 12 of 287 neurons) had an excitatory response to one tone and an inhibitory response to the other. Responses to cues had an average latency of $128.2 \pm 3.4 \mathrm{~ms}$ and reached a peak on average at $167.0 \pm 3.7 \mathrm{~ms}$. The peak of excitation was $9.0 \pm 0.9 \mathrm{~Hz}$ (corresponding to $0.615 \pm 0.007$ when firing rates were normalized, auROC); the peak of inhibition was $-4.0 \pm$ $0.4 \mathrm{~Hz}$ (corresponding to $0.426 \pm 0.005$ auROC; auROC normalization results in values ranging from 0 to 1 , with a value of 0.5 corresponding to baseline, and values $>0.5$ or $<0.5$ being excitatory or inhibitory responses respectively; Cohen et al., 2012).

To determine the overall ability of cue responses to differentiate between cues, the absolute difference in auROCs $(\triangle \mathrm{PSTH})$ between responses to Suc_cue and Q_cue was computed and averaged across cells. As shown in Figure $2 C$, the $\triangle \mathrm{PSTH}$ became significant in the first $50 \mathrm{~ms}$ bin $(0.017 \pm 0.003 \Delta$ auROC, corresponding to $0.98 \pm$ $0.28 \mathrm{~Hz} ; n=287$ cue-responsive neurons; $p<0.05$, Wilcoxon signed-rank test). The difference between responses to the two cues continued to increase over time, reaching a peak of $0.069 \pm 0.006$ $\triangle$ auROC (corresponding to $4.9 \pm 0.63 \mathrm{~Hz}$ ) at the end of the $250 \mathrm{~ms}$ window in which cue responses were examined.

Cue selectivity was then analyzed for each recorded neurons. Only $14.0 \%$ of cue-responsive neurons (40 of 287 neurons) responded indistinguishably to both cues (Fig. 2D, black bar). The majority of cue-responsive neurons either responded exclusively to one of the cues or had a significantly different response across cues ( $86 \%, 247$ of 287 neurons). Specifically, $16.0 \%$ of the neurons (46 of 287 neurons) responded to both cues, but differently (Fig. 2D, magenta bar), while $70.0 \%$ (201 of 287 neurons) responded exclusively to one of the two cues (i.e., cue selective; Fig. $2 D$, cyan and gold bars). As shown in Figure $2 D$, the majority of cue-responsive neurons $(55.0 \%, 158$ of 287 neurons) were activated exclusively by Suc_cue (vs $15.0 \%, 43$ of 287 neurons, responding exclusively to Q_cue). The majority of the cue responses were excitatory. To further quantify the selectivity of cue responses, the normalized $\triangle \mathrm{PSTH}$ was averaged across the first $250 \mathrm{~ms}$ for the different groups of neurons (Fig. 2E). This interval was chosen for consistency with previous results (Samuelsen et al., 2012) and because the probability of lever pressing was low in the first $250 \mathrm{~ms}$ and dramatically increased after that (occasional trials with faster lever presses were removed). Analysis of normalized $\triangle$ PSTH showed that neurons responding indistinguishably to both cues had a significantly lower $\triangle \mathrm{PSTH}$ than cue-specific neurons $(0.010 \pm 0.005 n=40$ responding to both cues vs $0.051 \pm 0.007, n=247$ responding to specific cue; $p<$ 0.01 , Wilcoxon rank-sum test). Altogether these results show that neurons in GC can respond selectively to cues anticipating different outcomes.

Visual inspection of the representative examples in Figure $2 \mathrm{~A}$ suggests that cue responses precede mouth movements triggered by the cue. Frame-by-frame automated video coding of mouth movements was performed on multiple sessions to exclude the 
possibility that cue-selective responses were secondary to mouth movements. Figure 3 features time course and latency analysis of mouth movements in relation to Suc_cue and Q_cue responses for a subset of randomly chosen sessions $(n=$ $104 ; 37.7 \%$ of the total number of go/ no-go sessions; 104 of 276 sessions). Of these 104 sessions, 47 contained cueresponsive neurons, accounting for $30 \%$ of the total number of cue-responsive neurons ( 87 of 287 neurons). In the first $250 \mathrm{~ms}$ following the cue, cues evoked mouth movements whose amplitude was dramatically smaller than that evoked by Suc (Suc_cue and Q_cue evoke mouth movements that are, respectively, 3.24\% and $8.04 \%$ of maximum movements evoked by Suc; $n=104$ sessions analyzed). The average onset of the first detectable mouth movement was at $348.6 \pm$ $19.1 \mathrm{~ms}$ for Suc_cue trials and 538.4 \pm 9.7 ms for Q_cue trials, both significantly slower than the latency of the respective cue responses recorded for this subset of sessions in GC (Fig. 3B; Suc_cue: $117.3 \pm$ $8.4 \mathrm{~ms}, n=48, p<0.01$; and Q_cue: $100.0 \pm 8.0 \mathrm{~ms}, n=29 ; p<0.01$, Wilcoxon signed-rank test; no significant difference was observed in the latency of responses to the two cues; $p>0.05$, Wilcoxon rank-sum test). To further confirm that mouth movements were not the major determinant of cue responses, the activity of cue-responsive neurons was aligned to the onset of the first mouth movement (data not shown). Inspection of population activity revealed that the largest modulation of firing relative to baseline preceded mouth movements. These results demonstrate that neural responses to cues consistently precede the onset of mouth movements.

Mouth movements are not the only possible motor confound in the go/no-go task; in go-trials (i.e., Suc_cue trials), animals press the lever to self-administer Suc. To determine whether cue specificity as well as the high number of neurons selective for Suc_cue were related to lever pressing, we analyzed how lever presses during the waiting time (i.e., uncued and unrewarded "ITI presses") changed the activity of cue-responsive neurons. To achieve sufficient statistical power, the analysis of neural activity evoked by ITI presses was performed only in sessions in which there were more than eight ITI presses $(n=87$ sessions with Suc_cue-responsive neurons and with at least eight lever presses during the ITI). Figure 4 features the results of this analysis. Lever presses that occurred during the waiting period and before the cue were associated with changes in spiking activity that were dramatically smaller than those observed in the case of
A

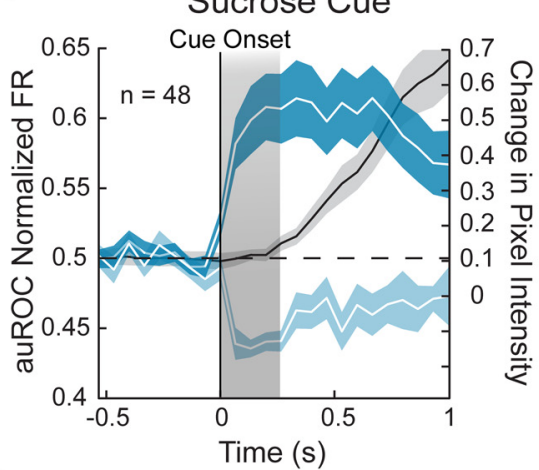

B

Sucrose Cue

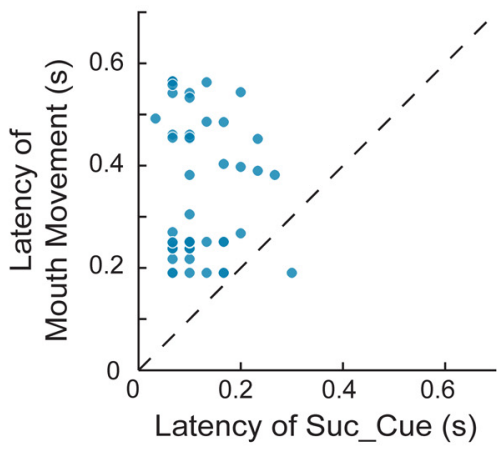

C

Sucrose
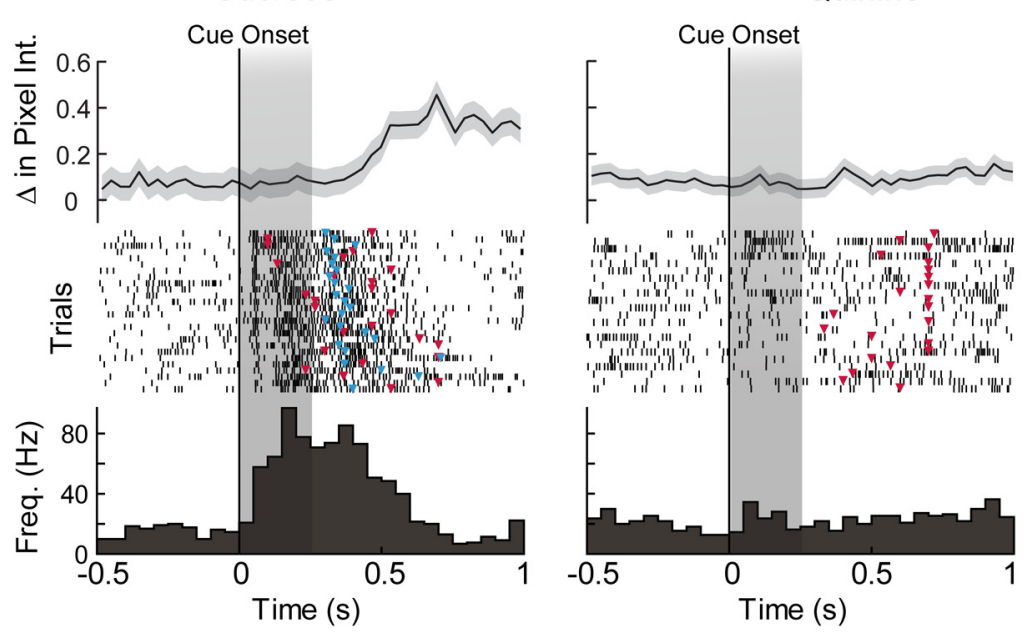

Figure 3. Cue responses precede mouth movements. A, Population PSTH for a subset of Suc_cue-selective (left, cyan traces) and Q_cue-selective (right, gold traces) neurons overlaid with the average time course of mouth movements as detected with a frame-by-frame image analysis (gray traces). Shading around traces, SEM; $x$-axis, time ( 0 , cue onset); $y$-axis for popPSTHs, auROC; $y$-axis for mouth movements, difference in pixel intensity for adjacent pixels. $\boldsymbol{B}$, Scatter plot comparing the latency of cue responses for individual neurons ( $x$-axis) with the latency of the earliest mouth movement detected in each session ( $y$-axis). Left, Suc_cue;

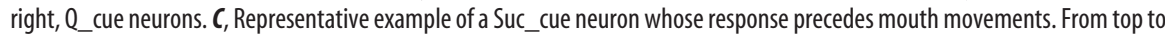
bottom: time course of mouth movements averaged for all the Suc_cue trials; raster plots and PSTHs for a single Suc_cue-selective neuron in response to Suc_cue (left) and $Q_{\text {_cue }}$ (right). $x$-axis, Time $(0$, cue onset); $y$-axis for mouth movements, difference of intensity for adjacent pixels; $y$-axis for rasters, trials; $y$-axis for PSTHs, firing frequency. Vertical line at time 0 indicates the onset of the cue. Cyan triangle superimposed to raster plots, Time of lever press; red triangle, time of earliest mouth movement detected. Shaded vertical rectangles on PSTHs show the time window in which cue responsiveness was considered.

cued lever presses. Activity was measured in the last bin before lever pressing. Neurons with selective Suc_cue responses showed significantly higher activity for cued lever presses compared with ITI presses (auROC excitatory response: $0.598 \pm 0.012$ for cued lever press vs $0.518 \pm 0.006$ for ITI press; $n=98 ; p<0.01$, Wilcoxon signed-rank test; auROC inhibitory responses: $0.429 \pm$ 

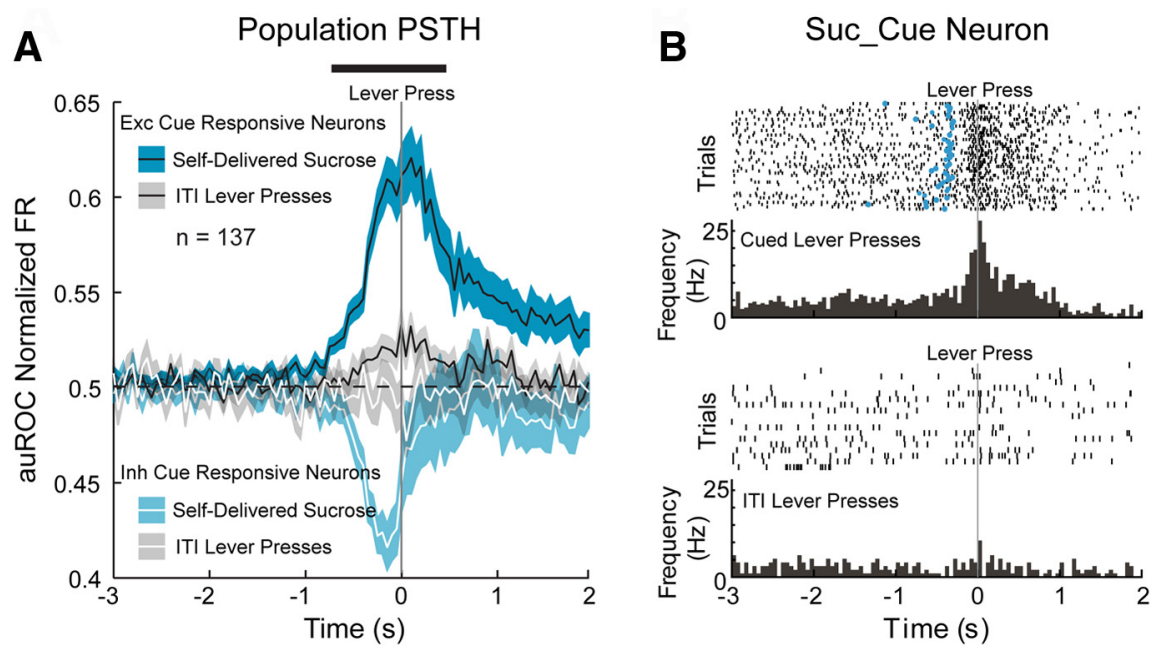

Figure 4. Suc_cue responses are not related to the motor act of lever pressing. A, Population PSTH for Suc_cue neurons in response to self-administrations (cyan traces) and to erroneous lever presses (gray traces). Erroneous lever presses are defined as those that occur in the absence of the cue and that do not lead to a Suc self-delivery. Shading around traces, SEM; $x$-axis, time $(0$, time at which lever is pressed); $y$-axis: auROC; thick line above traces, time interval in which popPSTH for self-delivery trials is significantly different from popPSTH for erroneous trials (excitatory and inhibitory responses combined). $\boldsymbol{B}$, Response to cued lever presses and erroneous lever presses in a representative Suc_cue-selective neuron. Top, Raster plot; bottom, PSTH; $x$-axis, time (vertical line at time 0 , time at which lever is pressed); $y$-axis for rasters, trials; $y$-axis for PSTHs, firing frequency; cyan marker superimposed on raster plots, onset of the cue.

0.014 for cued lever press vs $0.498 \pm 0.011 ; n=39$ for ITI press; $p<0.01$, Wilcoxon signed-rank test). A linear regression analysis of prepress activity between ITI presses and cued lever presses revealed a very small, albeit significant ( $p<0.01$, bootstrap), correlation $\left(R^{2}=0.183\right)$, confirming the weak influence of ITI presses on neural activity.

It is possible that, despite not being strongly modulated by ITI presses, cue-responsive neurons might be driven by a cuetriggered intention of pressing the lever. To determine the extent to which cue specificity was independent of cued motor action, a separate set of experiments was performed on a different cohort of animals. These experiments relied on pairing cues with passive deliveries of Suc and Q; a Suc_cue was followed by passive delivery of Suc, and a Q_cue was followed by passive delivery of Q. No lever was included in this paradigm, and no motor act was required. Analysis of neural activity triggered by the cue revealed that $22.9 \%$ of the neurons ( 25 of 109 neurons) were cue responsive. Of these, $32 \%$ of neurons ( 8 of 25 neurons) responded exclusively to the Suc_cue; $24 \%$ (6 of 25 neurons) responded exclusively to the Q_cue; $24 \%$ (6 of 25 neurons) responded to both cues, but differentially; and 20\% (5 of 25 neurons) responded to both cues indistinguishably. While the distribution of these cue responses was different from the one observed in the case of instrumental conditioning (see Discussion), the unequivocal presence of cue-specific responses indicates that cue specificity does not require cued lever pressing.

\section{Cue responses before and during learning}

To investigate whether cue responses depended on learning and how they varied with changes in the predictive value of the cue, cue-evoked activity was investigated in the following three additional conditions: (1) before rats learned to correctly perform the go/no-go task (no_learn sessions); (2) in the first sessions in which animals showed learning (first_learn sessions); and (3) after partial extinction of the cue-taste contingencies (partial_ext sessions).
First, we analyzed neural activity in the first go/no-go training session for animals that did not learn during this session (12 animals, 12 sessions, 95 neurons). Before the first session in which the two cues were introduced, rats had been trained on a single-cue, self-delivery paradigm (the single cue was a different tone from the two used for go/no-go; see Materials and Methods). Analyses were performed to determine the levels of baseline cue responsiveness in animals naive to the two cues. Figure $5 A$, left, shows a representative behavioral record for a first session in which the animal pressed in response to both cues in similar proportion, hence showing no sign of learning. The average best performance in the no_learn sessions was $65.8 \pm 3.0 \%$, with no sessions significantly differing from chance $(p>0.05$; see Materials and Methods). A total of 95 neurons was recorded in these conditions, and their cue responsiveness was analyzed. As expected from the prior training on one cue, $24.2 \%$ of the neurons ( 23 of 95 neurons) showed significant cue responses, the mean magnitude of which, relative to baseline, was comparable to that observed in well trained animals $(0.148 \pm 0.015$ auROC). The distribution of cue responses was analyzed and revealed significant differences when compared with the distribution observed in well trained animals. As shown in Figure $5 A$, middle, the majority of cue-responsive neurons (69.6\%, 16 of 23 neurons) responded nonselectively to both cues, and no Suc_cue-selective response was observed before the rat learning. This distribution was significantly different from the one observed after learning in well trained animals and featured in Figure $2 D(p<0.01,2 \times 4$ Fisher's exact test). To further verify the relative lack of selectivity of cue responses in the first session, the $\triangle$ PSTHs between cue responses was computed (Fig. $5 A$, right). The $\Delta$ PSTH values averaged over $250 \mathrm{~ms}$ following the cue showed few signs of cue specificity compared with baseline $(0.019 \pm 0.009 \Delta$ auROC; $n=23$ cue-responsive neurons; $p>0.05$, Wilcoxon signed-rank test) and were significantly lower than the ones computed in well trained animals (Figs. 2C, $5 A$, right, dotted trace; $0.091 \pm 0.007 \Delta$ auROC; $n=287$ cueresponsive neurons; $p<0.01$, Wilcoxon rank-sum test). Altogether, these results show that cue responses before learning the go/no-go task lack the selectivity observed after learning.

To track the possible emergence of cue selectivity, we analyzed cue responses in a subset of animals that went from unbiased pressing (i.e., $50 \pm 10 \%$ correct performance) to criterion performance within a single session during either the first or the second training day. A total of 18 sessions was included in this analysis ( 18 rats, 108 neurons; in 5 of the 23 sessions, the animal demonstrated biased behavior from the beginning of the session and so were not included in the analysis, unless specified). Figure $5 B$, left, shows an example of a first session in which the rat shows learning. Rats in this group reached criterion performance after $30.3 \pm 9.1$ trials, with all sessions significantly differing from chance performance (see Materials and Methods). A total of 35 cue-responsive neurons was found in these sessions. Analysis of the distribution of cue-responsive neurons (Fig. 5B, middle) showed a significant bias toward cue-selective responses com- 
A No_Learn Session

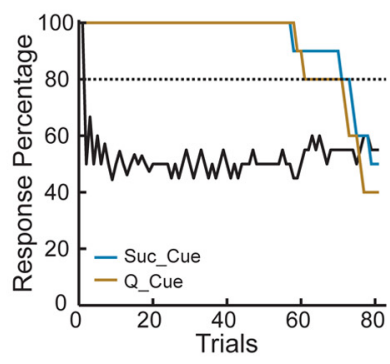

B

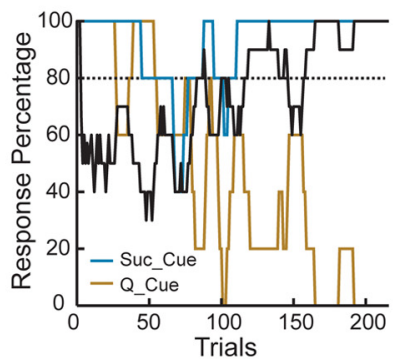

C Within Session Performance

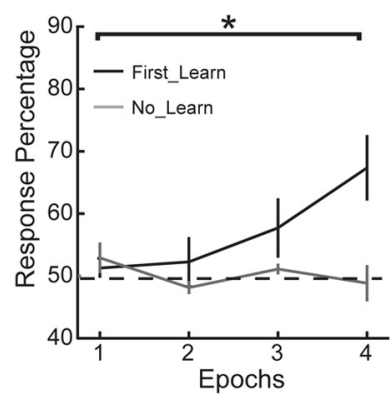

Distribution of Cue Responses No_Learn Sessions

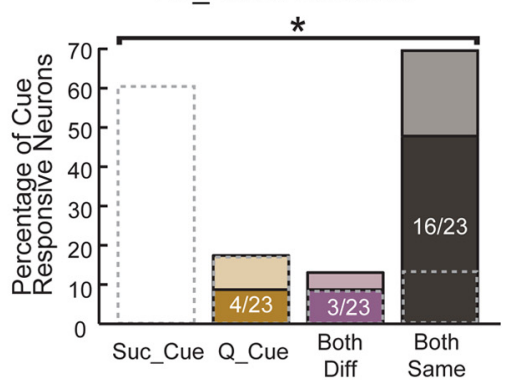

Distribution of Cue Responses First_Learn Sessions

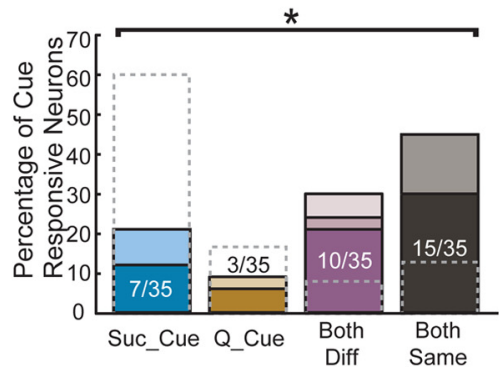

Within Session Cue Selectivity
No_Learn vs. Well-Trained

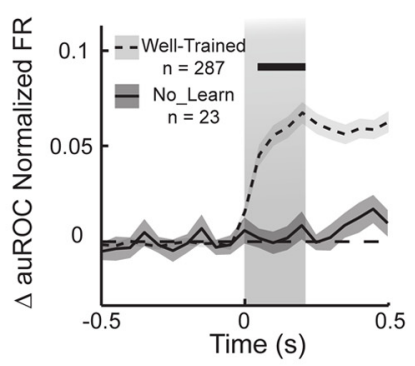

$\triangle \mathrm{PSTH}$

First_Learn vs. Well-Trained

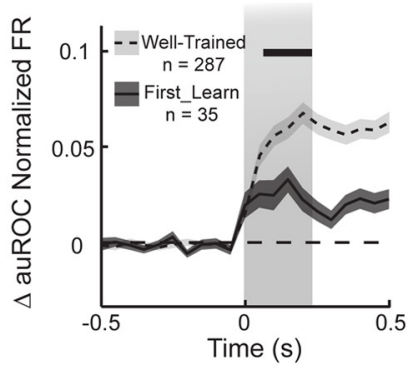

Whithin Session Emergence of Cue Selecivity

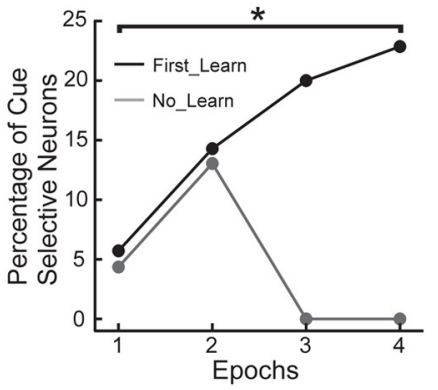

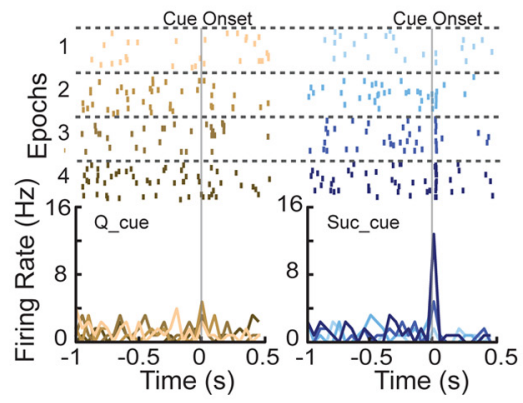

Figure 5. Cue-specific responses develop with learning. $\boldsymbol{A}$, Cue responses in first go/no-go training sessions for rats that do not learn in the first session (i.e., no_learn sessions). Left, Representative behavioral record of a first go/no-go training session; $x$-axis, trials; $y$-axis, percentage of cues followed by a lever press; cyan line, Suc trials; gold line, $Q$ trials; black line, performance averaged across cues; dashed line, criterion level. Middle, Distribution of cue-responsive neurons recorded in no_learn sessions. Gray dashed bars, Distribution of cue responses in well trained rats [Suc_cue-selective (cyan), Q_cue-selective (gold), differentially responding to both cues (magenta), equally responding to both cues (black)]. Dark shades, Excitatory responses; light shades, Inhibitory responses. Right, Absolute difference between PSTHs in response to Suc_cue and Q_cue averaged across neurons. $x$-axis, time (0, cue onset); $y$-axis, $\Delta$ auROC; solid line, no_learn; dashed line; well trained animals; shading around traces, SEM; thick line above traces, time interval in which popPSTH for no_learn sessions is significantly different from popPSTH for well trained sessions.

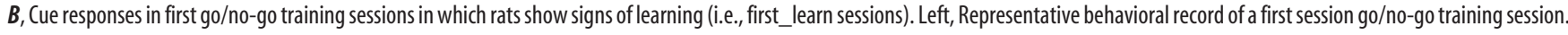
Middle, Distribution of cue-responsive neurons recorded in first_learn sessions. Right, Absolute difference between PSTHs in response to Suc_cue and Q_cue averaged across neurons. Conventions are as in $\boldsymbol{A}$. C, Within-session analysis of learning. Left, Correct performance for each quarter of no_learn (light gray) and first_learn (dark gray) sessions. Middle, Percentage of cue-responsive neurons that respond selectively to cues for each quarter of no_learn (light gray) and first_learn (dark gray) sessions. Right, Representative neuron developing cue selectivity within a single first_learn session. Top, Raster plots divided by quarters; bottom, PSTHs for each quarter; blue lines, Suc trials; gold lines, $Q$ trials; $x$-axis, time (vertical line at time 0 , cue onset); $y$-axis for rasters, trials; $y$-axis for PSTHs, firing frequency. Asterisks indicate significant differences.

pared with no_learn sessions $(p<0.05,2 \times 4$ Fisher's exact test). In first_learn sessions, $57.1 \%$ of the cue-responsive neurons (20 of 35 neurons) differentiated between cues, whereas only $30.4 \%$ of cue-responsive neurons ( 7 of 23 neurons) were cue differentiating in no_learn sessions. Analysis of the average $\triangle$ PSTHs (Fig. $5 B$, right) further confirmed the presence of cue selectivity in first_learn sessions: while $\triangle$ PSTH in no_learn sessions was not significantly different from baseline (see above), the $\triangle \mathrm{PSTH}$ in first_learn was significantly larger than that at baseline $(0.037 \pm$ $0.012 \Delta$ auROC; $n=35$ cue-responsive neurons; $p<0.05$, Wilcoxon signed-rank test). Interestingly, the $\triangle \mathrm{PSTH}$ in first_learn was not as large as in well trained animals $(0.091 \pm 0.007$
$\Delta$ auROC; $n=287$ cue-responsive neurons; $p<0.01$, Wilcoxon rank-sum test).

To track both learning and the parallel development of cue responses, no_learn and first_learn sessions were divided into four epochs, each containing the same number of trials. Figure $5 C$, left, shows the behavioral performance in each of the four epochs for no_learn sessions (light gray; $n=6$ sessions) and for first_learn sessions (black; $n=12$ sessions) in which cueresponsive neurons were recorded. Performance remained at a chance level throughout the session in the case of no learning, and progressively increased in successive epochs for sessions in which animals learned. The selectivity of cue responses was compared in 
A

Partial Extinction Session

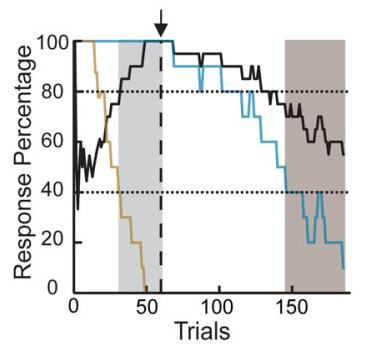

\section{Population PSTH} Pre- and Post-Extinction

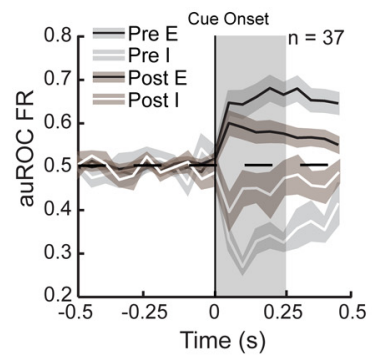

B
C Amplitude
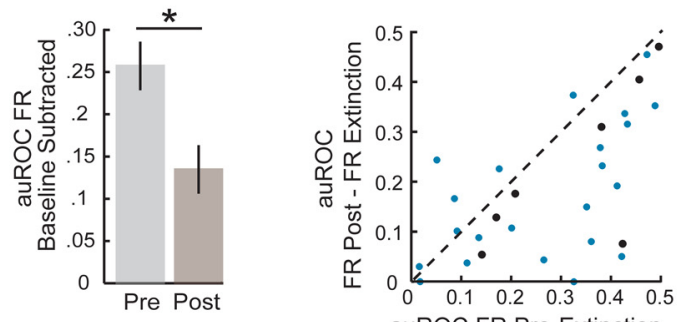

E

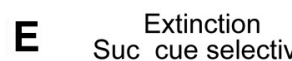
Suc_cue selective Sucrose Cue Quinine Cue

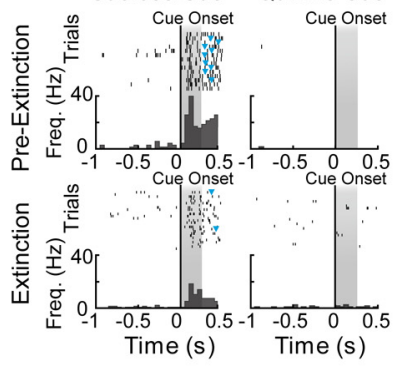
auROC FR Pre-Extinction

Cue Responses Pre- and Post-Extinction

\section{Cue Responses During
Mid vs Late Session}

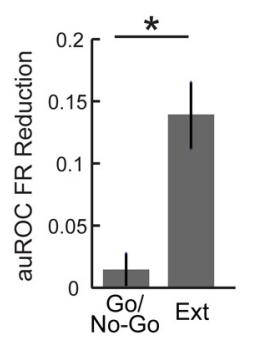

Figure 6. Magnitude of cue responses is significantly reduced by extinction. $\boldsymbol{A}$, Representative behavioral record of a partial extinction session. Cyan line, Suc trials; gold line, Q trials; black line, correct performance averaged across cues; light gray shading, time period in which behavior was above criterion performance; arrow, beginning of partial extinction; dark gray shading, time period with partially extinguished performance; top dashed line, criterion level; bottom dashed line, partial extinction level ( $<40 \%$ of pressing to Suc_cue). B, Magnitude of Suc_cue responses before (i.e., at criterion performance) and after partial extinction. Asterisk indicates significant differences. C, Scatter plot showing cue responses before and after partial extinction for each cue-responsive neuron. A total of $78.4 \%$ of the neurons ( 29 of 37 neurons) shows smaller responses after extinction. Dark blue dots, Nonselective neurons; light blue dots, Suc_cue-selective neurons. D, Population PSTHs for excitatory (black lines) and inhibitory (white line) responses before (light gray shading) and after (dark gray shading) partial extinction. Pre E, Excitatory responses before extinction; Pre I, inhibitory responses before extinction; Post E, excitatory responses after extinction; Post I, inhibitory responses after extinction; $x$-axis, time ( 0 , cue onset); $y$-axis, auROC; shading around traces, SEM. $E$, Representative raster plots and PSTHs in response to Suc_cue (left) and Q_cue (right) before (top) and after (bottom) extinction. $x$-axis, Time (vertical line at time 0 , cue onset); $y$-axis for rasters, trials; $y$-axis for PSTHs, firing frequency; cyan marker, time of self-delivery. $\boldsymbol{F}$, Reduction of cue responses as result of time passing in a regular go/no-go session (left bar) and in length-matched extinction sessions. Error bars represent the SEM. Asterisk indicates significant differences.

these two types of sessions, as detailed in Figure $2 C$, middle. No_learn and first_learn sessions showed a similar number of neurons differentiating the two cues in the first two epochs (epoch 1: no learning, 4.4\%, 1 of 23 neurons; vs learning, 5.7\%, 2 of 35 neurons; $p>0.05$; epoch 2: no learning, $13.0 \%, 3$ of 23 neurons, vs learning, $14.3 \%, 5$ of 35 neurons; $p>0.05$ ). However, in the third and fourth epoch, those in which learning began to appear, the situation dramatically changed. While the number of neurons differentiating between the two cues dropped to zero for no_learn sessions, it continued to increase for first_learn sessions, reaching a maximum of $22.9 \%$ ( 8 of 35 cue-responsive neurons; $p<0.05$ for the third and fourth epoch, Fisher's exact test) of the cue-responsive neurons, which significantly increased from the first epoch of the learning sessions $(p<0.05$, one-sided $\mathrm{McNemar}$ test). The representative neuron featured in Figure 5C, right, shows the progressive development of a Suc_cue-selective response.

\section{Cue responses after partial extinction}

To further determine how cue responses varied, depending on the behavioral significance of the cue, 27 partial extinction sessions were performed in 13 rats (partial extinctions were repeated upon complete relearning of the task). A total of 127 neurons was

recorded in this condition; 41 neurons were cue responsive, 32 of which were cue selective (28 for Suc_cue and 4 for Q_cue). Well trained rats entered a regular go/no-go test session, and after reaching and maintaining the criterion performance for a minimum of 30 trials, the contingencies were extinguished, and cues were no longer followed by the availability of Suc or Q. As shown in Figure 6A, which features a representative partial extinction session, lever pressing for Suc_cue began to decline soon after the beginning of extinction. Partial extinction was defined as a decrease to $\leq 40 \%$ of correct responses for the Suc_cue. Spontaneous and cue-evoked activity of neurons that preferentially responded to the Suc_cue was quantified during the period of performance above criterion before the beginning of extinction (Fig. 6A, light gray shading) and after achieving partial extinction (Fig. 6A, dark gray shading). The magnitude relative to baseline of excitatory and inhibitory cue responses to the Suc_cue were significantly reduced by partial extinction, as they dropped from an average value of $0.268 \pm 0.025$ to $0.146 \pm 0.027$ auROC $(n=37 ; p<0.01$, Wilcoxon signed-rank test). The reduction in the normalized response was not related to an increase in baseline firing; in fact, a small, yet significant, reduction in background activity was observed with extinction (from $13.0 \pm 1.9$ to $11.1 \pm 1.5$ $\mathrm{Hz} ; p<0.01$, Wilcoxon signed-rank test). Figure 6 shows the significant reduction in Suc_cue-evoked activity averaged across Suc_cue-specific cells (Fig. 6B) or for each individual neuron (Fig. 6C). As shown in Figure $6 \mathrm{C}$, the vast majority of the neurons responding to Suc_cue exhibited lower activity after partial extinction (Suc_cue responsive neurons, $78.4 \%$, 29 of 37 neurons; $p<0.01$, sign test). The subset of Suc_cue-selective neurons $(n=28)$ showed a very similar percentage reduction (blue circles; $75.0 \%, 21$ of 28 neurons; $p<0.01$, sign test). A significant reduction of cue responses following partial extinction could also be observed by comparing popPSTH for excitatory and inhibitory responses (Fig. 6D). Both types of responses decreased after extinction, and the reduction lasted for the entire $250 \mathrm{~ms}$ window examined. The reduction was significant for excitatory responses (average response range, $0.746 \pm 0.035$ to $0.639 \pm 0.032$ auROC; $n=32 ; p<$ 0.01 , one-sided Wilcoxon signed-rank test) and trending due to the small number of inhibitory responses (average response range, $0.197 \pm 0.019$ to $0.387 \pm 0.071$ auROC; $n=5 ; p=0.06$, one-sided Wilcoxon signed-rank test). Visual inspection of raster plots and PSTHs for a representative neuron confirms a clear reduction of the firing activity evoked by the Suc_cue after partial extinction (Fig. $6 E$ ). Figure $6 F$ shows the results of a control performed to verify that the partial extinction of cue responses was not just an effect of time passing. Cue responses were analyzed over the duration of regular go/no-go sessions. Suc_cue responses in the early postcriterion period (1-10 tri- 
A
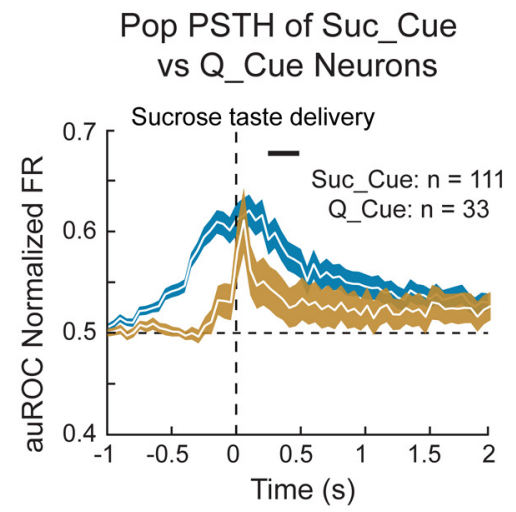

B

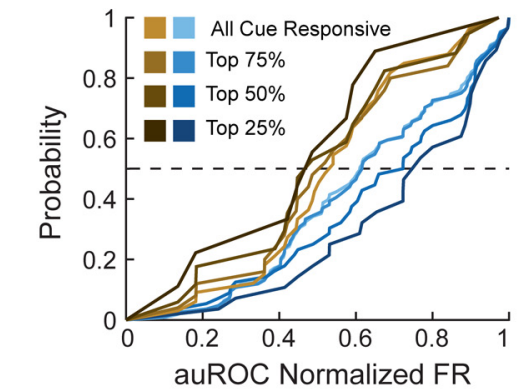

Cumulative Distribution Function

Quinine Cue
C

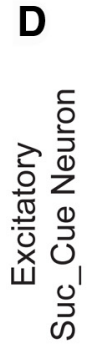
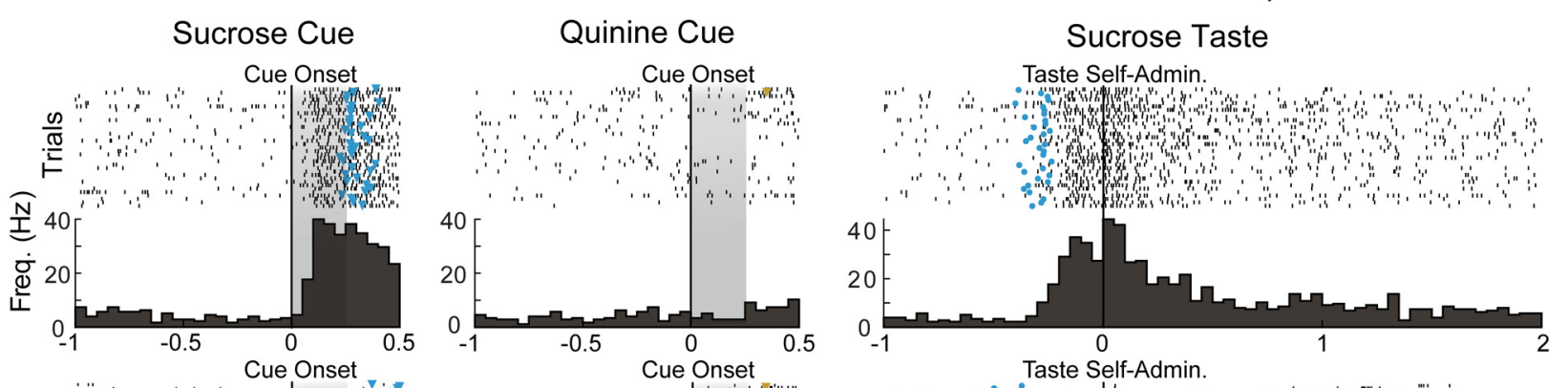

Sucrose Taste

Response to Sucrose

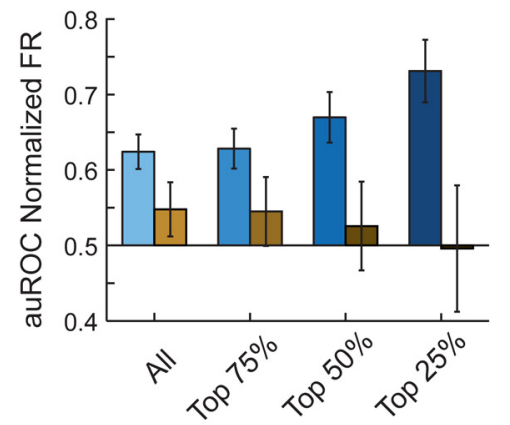

Taste Self-Admin.

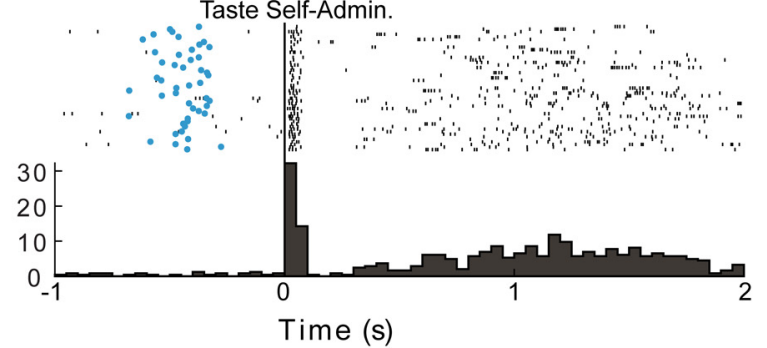

Figure 7. Suc_cue neurons have larger responses to sucrose compared with Q_cue-selective neurons. $\boldsymbol{A}$, Population PSTH of excited Suc_cue-selective (cyan) and Q_cue-selective (gold) neurons in response to Suc self-administration. $x$-axis, Time ( 0 , Suc delivery); $y$-axis, auROC; shading around traces, SEM; horizontal bar above traces, time interval in which responses are significantly different. $\boldsymbol{B}$, Cumulative distribution function of responses to Suc for Suc_cue-selective (shades of blue) and Q_cue-selective (shades of gold) neurons grouped according to the strength of the cue response. $x$-Axis, auROC; $y$-axis, probability. $C$, Responses to Suc averaged between 250 and 500 ms and across different groups of Suc_cue-selective (shades of blue) and $Q$ _cue-selective (shades of gold) neurons. $x$-axis, Groups of cue-responsive neurons according to strength of response; $y$-axis, auROC; error bars, SEM. $D$, Representative examples of a neuron selective to the Suc_cue (top row) and one selective for the $\mathbf{Q} \_$cue (bottom row). Raster plots (top) and PSTHs show responses to the Suc_cue (left), $Q$ _cue (middle), and Suc taste (right). $x$-Axis, Time (vertical line at time 0 in Suc_cue and Q_cue rasters and PSTHs, onset of cue; vertical line at time 0 in Suc taste rasters and PSTHs, time of Suc self-delivery); $y$-axis for rasters, trials; $y$-axis for PSTHs, firing frequency; cyan triangle, time of Suc self-delivery; gold triangle, time of Q self-delivery; cyan circle, time of cue onset.

als after reaching criterion) were compared with responses for the same neurons in the late postcriterion period (31-40 trials after reaching criterion). The same analysis was performed for a time-matched interval in extinction sessions. The absence of significant differences in regular sessions $(0.015 \pm 0.013$ $\Delta$ auROC; $n=113$ Suc_cue-responsive neurons; $p>0.05$, Wilcoxon signed-rank test) ruled out the effects of time on the reduction of cue responses within a session. Results from the equivalent test in time-matched extinction sessions $(0.138 \pm$ $0.027 \Delta$ auROC; $n=31$ Suc_cue-responsive neurons; $p<0.01$, Wilcoxon signed-rank test) confirmed that the reduction of cue responses observed in partial extinction sessions was linked to the change in predictive value of the cue. Comparison of the $\triangle$ auROC in time-matched control and extinction revealed a significant difference $(p<0.01$, Wilcoxon ranksum test).

Altogether, the results outlined above clearly indicate a direct relationship between cue responsiveness in GC and the expectation of a specific outcome.

\section{Relationship between cue and sucrose taste responsiveness}

Models of sensory processing have suggested that expectation might result in the anticipatory activation of stimulus-specific representations (Rao and Ballard, 1999; Summerfield and Egner, 2009; Zelano et al., 2011). A series of analyses was performed on cue-responsive neurons to determine how the responsiveness of a neuron to a specific cue related to its response to taste. Analyses of taste responses were limited to Suc since correct learning of the task implied no pressing for Q (hence minimal Q trials). Figure $7 A$ shows popPSTH in responses to Suc for neurons excited selectively by Suc_cue $(n=111$, cyan) and Q_cue $(n=33$, gold $)$. Suc_cue neurons produced significantly larger responses to Suc_cue than Q_cue neurons ( $p<0.01$, two-way ANOVA; 250 ms bins; main effect of taste condition), with the largest difference occurring in the 250-500 ms interval. Mouth movements were analyzed for sessions in which Suc_cue and Q_cue neurons were recorded to determine whether the difference in response to Suc between 250 and 500 ms was related to movements. Within the 250-500 ms interval, mouth movements in response to Suc 
were nearly identical ( $p>0.05$, Wilcoxon rank-sum test) for trials in which Suc_cue neurons $(0.73 \pm 0.09, n=29$ sessions $)$ and Q_cue neurons ( $0.72 \pm 0.11, n=22$ sessions) were recorded. This similarity excludes movement as a source of difference in Suc responses between Suc_cue- and Q_cue-responsive neurons. Additional analyses were performed to determine whether the difference in responsiveness to Suc depended on the strength of the selective cue response. Suc_cue- and Q_cue-responsive neurons were grouped according to the magnitude of their cue response. The cumulative distribution function (Fig. $7 B$ ) and the average magnitude of responses to Suc (Fig. 7C) were computed for the following groups of neurons selectively excited by either one of the two cues: all of the cue-responsive neurons regardless of the magnitude of the cue response; neurons having Suc_cue and Q_cue responses in the top 75\% of cue response magnitude; neurons having Suc_cue and Q_cue responses in the top 50\% of cue response magnitude; or neurons having Suc_cue and Q_cue responses in the top $25 \%$ of cue response magnitude. The separation of Suc responses between Suc_cue (cyan) and Q_cue (gold) neurons progressively increased with the strength of the cue response (Suc_cue vs Q_cue: all: $0.624 \pm 0.023$ vs $0.548 \pm$ $0.036 ; p=0.059$; top $75 \%$ : $0.628 \pm 0.026$ vs $0.545 \pm 0.046 ; p=$ 0.068 ; top $50 \%$ : $0.670 \pm 0.034$ vs $0.526 \pm 0.0059 ; p<0.05$; top $25 \%$ : $0.731 \pm 0.041$ vs $0.496 \pm 0.084 ; p<0.01)$. The response to Suc was the largest in neurons with the strongest response to Suc_cue and the smallest in neurons with the strongest response to Q_cue. The same analyses performed on neurons with inhibitory responses to Suc_cue and Q_cue yielded qualitatively and quantitatively similar results (data not shown). Figure $7 D$ shows representative examples of Suc_cue and Q_cue neurons displaying the behavior detailed above.

To further understand the relationship between cue and taste responsiveness, additional analyses were performed for the largest group of cue-responsive neurons (i.e., neurons responding selectively to Suc_cue). Figure 8 shows responses to Suc analyzed for the following three groups of neurons: neurons that produced excitatory Suc_cue responses, neurons that produced inhibitory Suc_cue responses, and neurons that were not cue responsive. Figure $8 A$ shows population responses to Suc for the three groups. At a population level, neurons that had excitatory responses to Suc_cue were also excited by Suc (Fig. 8A, left). On the contrary, the population of neurons that were inhibited by the Suc_cue showed inhibition in response to Suc (Fig. $8 A$, middle). Neurons that were not cue responsive showed a population response that was biased toward excitation, yet this excitation was significantly smaller than that evoked in neurons producing excitatory Suc_cue responses (Fig. 8A, right). Quantification of the average firing rates in the first $250 \mathrm{~ms}$ following gustatory stimulation (Fig. $8 B$ ) revealed that Suc evoked significantly higher firing in neurons excited by Suc_cue than in the other two groups (Suc_cue excitatory: 0.696 $\pm 0.023, n=111$; Suc_cue inhibitory: $0.431 \pm 0.036, n=47$; Suc_cue nonresponsive: $0.538 \pm 0.008$, $n=472 ; p<0.01$, Kruskal-Wallis test; all paired comparisons have $p<0.01$ in familywise corrected post hoc analysis). A $250 \mathrm{~ms}$ period was chosen for compatibility with the period of analysis of cue responses. Additional cell-by-cell analyses were performed to investigate how individual units encoding Suc_cue responded to Suc. Single-neuron responses to Suc were divided into excitatory, inhibitory, and nonresponsive on the basis of firing rates averaged over $250 \mathrm{~ms}$ following the self-administration of Suc. Figure $8 C$ shows the distribution of responses to Suc in cue-responsive and non-cue-responsive neurons. The majority of the neurons excited by the Suc_cue $(64.0 \%, 71$ of 111 neurons) showed excit- atory responses to Suc (matching neurons), hence reflecting the population average shown in Figure $8 A$, left. Only a minority of the neurons that were excited by Suc_cue showed mismatching responses to Suc: $16.2 \%$ (18 of 111 neurons) were inhibited by Suc, while $19.8 \%$ (22 of 111 neurons) were not responsive. A similar analysis was performed on neurons with inhibitory responses to Suc_cue. The majority of neurons inhibited by Suc_cue were inhibited by Suc (inhibited: 53.2\%, 25 of 47 neurons; excited: $25.5 \%, 12$ of 47 neurons; nonresponsive: $21.3 \%, 10$ of 47 neurons), confirming the overall predominance of matching responses $\left[p<0.01,3 \times 3 \chi^{2}\right.$ test for independence; all paired comparisons $\left(2 \times 3 \chi^{2}\right.$ tests with Sidak correction) have $p<$ $0.01]$. Interestingly, half of the neurons nonresponsive to the Suc_cue did not respond to Suc $(50.6 \%, 239$ of 472 non-cueresponsive neurons); the neurons in this group that responded to Suc showed a small bias toward excitation [29.2\% (138 of 472 neurons) vs $20.1 \%$ (95 of 472 neurons); $p<0.01, \chi^{2}$ test for independence]. The probability distribution (Fig. $8 C$, bottom right) and the counts of neurons in each group (Fig. $8 \mathrm{C}$, top right) confirm the bias toward matching responses in cue-responsive neurons.

Altogether, these data show that the majority of neurons that are selectively activated by Suc_cue have matching responses to the cue and taste.

\section{Matching neurons and responses to unexpected deliveries and omissions}

Additional experiments and analyses were performed to determine the functional role of matching neurons. Specifically, their involvement in coding different aspects of expectation was investigated. The first set of experiments tested whether matching neurons differentially encoded expected versus unexpected stimuli. Responses to surprising taste deliveries were investigated in sessions in which Suc was unexpectedly delivered during the waiting time preceding the onset of the cue (16 rats, 51 sessions, 302 neurons). The results of this experiment are featured in Figure 9. Responses of both groups of matching neurons (i.e., those that were excited by cue and taste, and those that were inhibited by cue and taste) to expected and unexpected Suc were analyzed.

A comparison of population responses in the first $250 \mathrm{~ms}$ revealed a significant, yet small, difference in the average magnitude of activity evoked by expected and unexpected Suc for matching excitatory and inhibitory responses. The magnitude relative to baseline of responses to expected Suc tended to be larger than to unexpected Suc $(0.341 \pm 0.024$ vs $0.269 \pm 0.033$ auROC with baseline subtracted; $n=22$ Suc_cue matching neurons; $p<0.05$, Wilcoxon signed-rank test). A cell-by-cell analysis revealed that $59.1 \%$ (13 of 22 Suc_cue-matching neurons) of the matching neurons had significantly different responses depending on whether the tastant was cued and self-delivered or uncued and automatically delivered. As shown in Figure 9B, the majority of neurons had only slightly stronger excitatory or inhibitory responses to expected Suc. While the difference was significant, it was small, and the overall ratio between responses to unexpected and expected Suc was only 0.719. Additional analysis showed that the difference was limited to the first $50 \mathrm{~ms}$, after which the similarity between responses in the two conditions increased (50250 ms bin: $0.321 \pm 0.026$ vs $0.282 \pm 0.034$ auROC, baseline subtracted; $n=22$ Suc_cue-matching neurons; $p>0.05$, Wilcoxon signed-rank test). Visual inspection of representative examples of matching cue-responsive neurons (Fig. 9C) confirms the results, showing a slightly, and transiently, stronger response to expected Suc and an increase of similarity after the initial bins. 
A

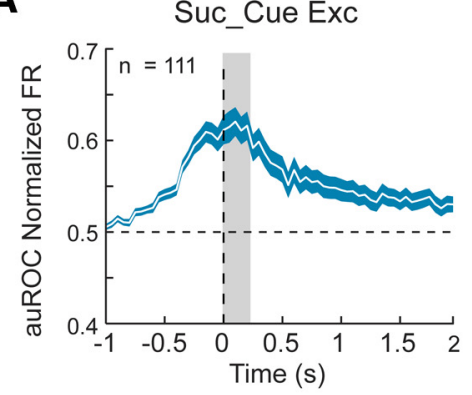

B
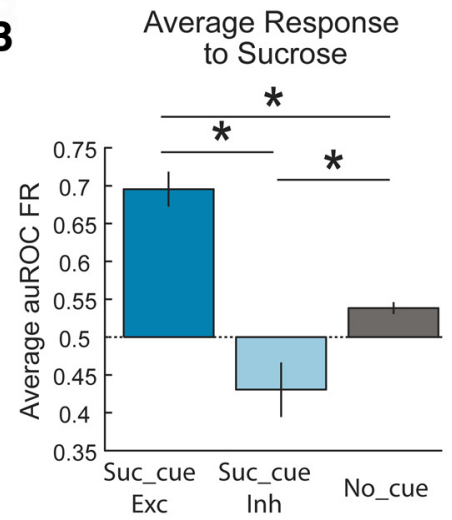

D
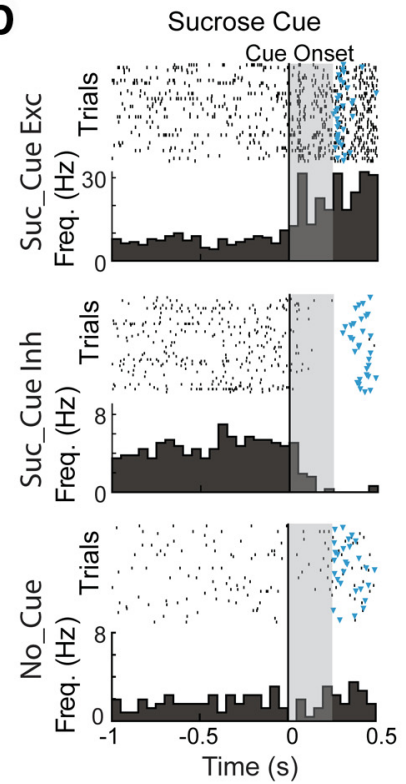

Suc_Cue Inh

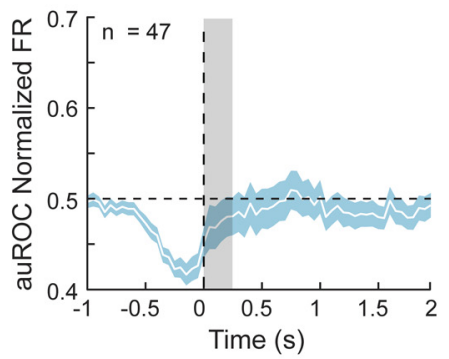

Histogram

C

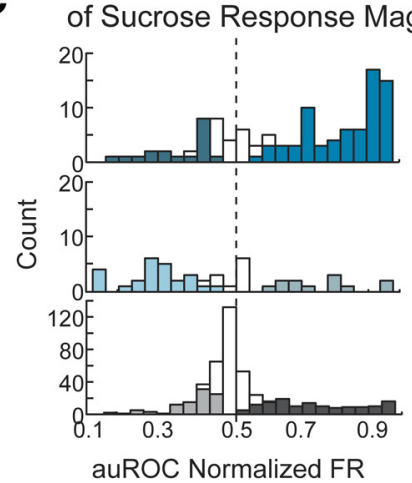

Non_Cue-Responsive

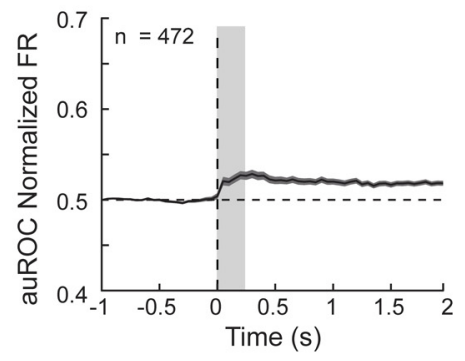

Percentage and CDFs of Sucrose Responses
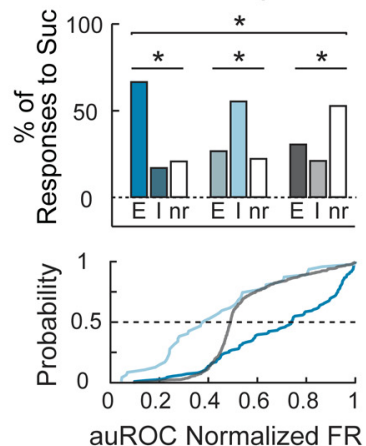
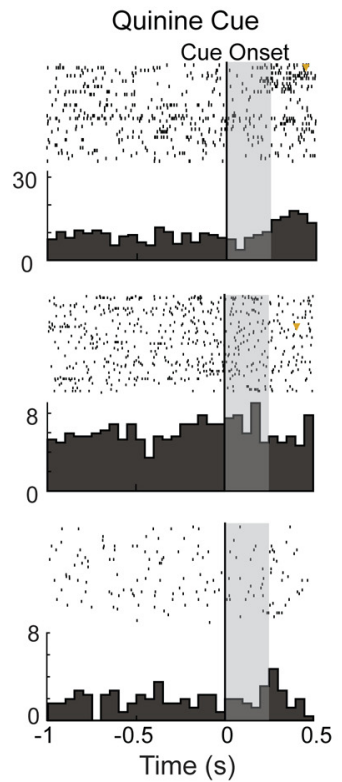

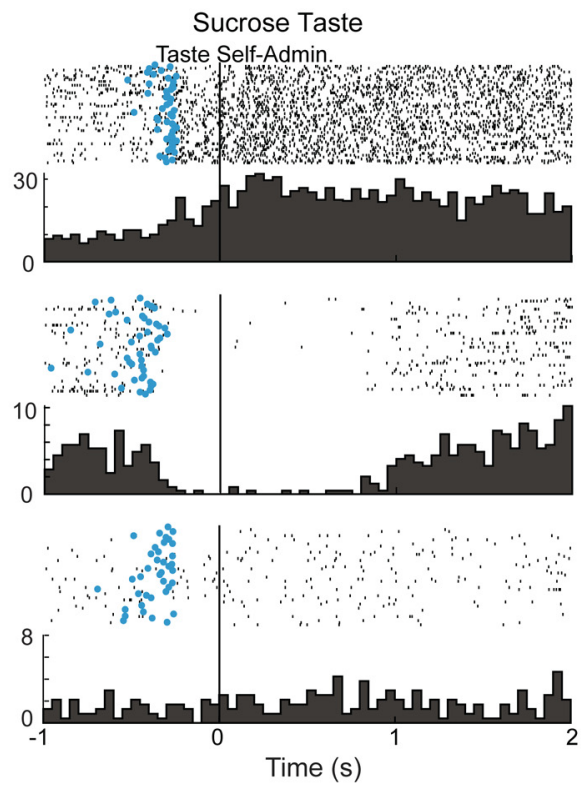

Figure 8. Responses to sucrose match responses to Suc_cue. A, Population PSTH in response to Suc for the following three groups of neurons: neurons with selective excitatory responses to Suc_cue (left, cyan); neurons with selective inhibitory responses to Suc_cue (middle, light blue); and neurons with no responses to either cue (right, gray). $x$-axis, Time (vertical dashed line at 0 , Suc delivery); $y$-axis, auROC; shading around traces, SEM; horizontal dashed line, baseline auROC. B, Suc responses averaged across neurons and over 250 ms following taste delivery in the three groups of neurons outlined in $\boldsymbol{A}$. Same color conventions as $\boldsymbol{A}$. Error bars represent the SEM. Asterisks indicate significant differences. $\boldsymbol{C}$, Left, Frequency histogram of responses to Suc for three groups of neurons. $x$-axis, auROC; $y$-axis, count; dark colors (i.e., dark blue, dark cyan, and dark gray), neurons with excitatory responses to Suc; light colors (i.e., light blue, light cyan, and light gray), neurons with inhibitory responses to Suc; white, neurons with no responses to Suc. Right, Top, Percentage of neurons with excitatory, inhibitory, and nonsignificant responses to Suc for each of the three groups described in $A$. $x$-axis, Subgroups; $y$-axis, percentage of total neurons responding to Suc. Right, Bottom, Cumulative distribution function for neurons with selective excitatory responses to Suc_cue (cyan), neurons with selective inhibitory responses to Suc_cue (light blue), neurons with no responses to either cue (gray). $x$-axis, auROC; $y$-axis, probability. Asterisks indicate significant differences. $D$, Representative raster plots and PSTHS for two matching neurons and a non-cue-responsive neuron. Top row, Neuron excited by Suc_cue and Suc; middle row, neuron inhibited by Suc-cue and Suc; bottom row, non-cue-responsive neuron; $x$-axis, time (vertical line at time 0 in Suc_cue and Q_cue rasters and PSTHs, onset of cue; vertical line at time 0 in Suc taste rasters and PSTHs, onset of Suc self-delivery); $y$-axis for rasters, trials; $y$-axis for PSTHs, firing frequency; cyan triangle, time of Suc self-delivery; gold triangle, time of Q self-delivery; cyan circle, time of Suc_cue onset.

While these results highlight a bias toward expected relative to unexpected Suc, the bias is very transient and small with the majority of neurons ( $77.2 \%, n=17$ of 22 neurons) being activated by both expected and unexpected Suc. This overall similar- ity between responses to expected and unexpected Suc is relevant, because it suggests that responses to expected Suc are not the result of cue-evoked activity carrying over into the taste delivery period. Indeed, visual inspection of the representative neuron in 
A

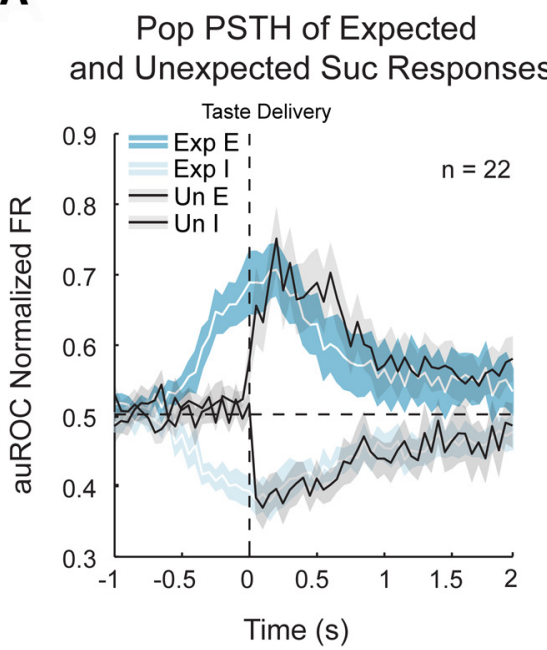

B
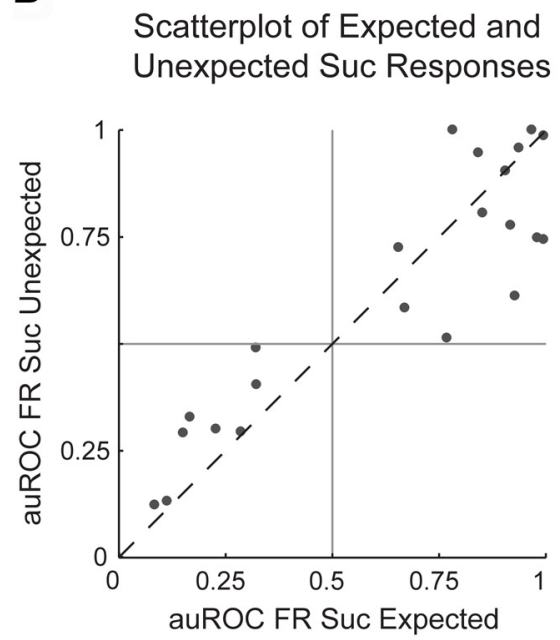

C

\section{Suc_Cue Matching Neuron}

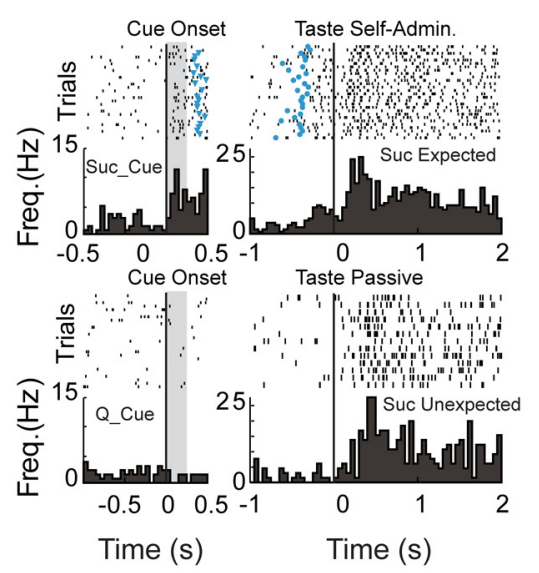

Figure 9. Matching neurons do not code prediction error. A, Pop PSTHs for matching neurons in responses to self-delivered Suc (cyan shading; Exp E, excitatory responses; Exp I, inhibitory responses) and unexpectedly delivered Suc (gray shading; Un E, excitatory responses; Un I, inhibitory responses). $x$-axis, Time (dashed line at 0 , Suc delivery); $y$-axis, auROC. Population PSTHs are plotted for both excitatory and inhibitory responses. Shading, SEM. B, Scatter plot showing responses to unexpected vs expected Suc. $x$-axis, auROC; $y$-axis, auROC. C, Representative raster plot and PSTHs for a Suc_cue-selective neuron showing responses to unexpected and expected Suc. $x$-axis, Time (vertical line at time 0 in left rasters and PSTHs, onset of cue; vertical line at time 0 in right rasters and PSTHs, onset of Suc delivery); $y$-axis for rasters, trials; $y$-axis for PSTHs, firing frequency; cyan triangle, time of Suc self-delivery; cyan circle, time of Suc_cue onset.

Figure $9 C$ confirms that little or no continuation of cue responses is observed in the taste period.

A second subset of experiments was designed to investigate how matching neurons responded when expectations were violated. Neurons were recorded in sessions in which, for random trials (20\% of Suc_cue trials), Suc was not delivered following the correct lever press to Suc_cue (omission). Responses to expected and omitted Suc were compared in 50 matching neurons (32 neurons with excitatory Suc_cue responses and 18 neurons with inhibitory Suc_cue responses) recorded during omission sessions (15 rats, 75 sessions, 419 neurons). Figure 10A, left, shows population activity of matching neurons evoked by Suc (cyan) and omissions (gray). Both excitatory (upward traces) and inhibitory (downward traces) matching responses were analyzed. Analysis of omission-evoked activity revealed that $74.0 \%$ (26 of 32 excitatory matching neurons; 11 of 18 inhibitory matching neurons) of matching neurons responded significantly to omissions. The population of neurons with excitatory matching activity $(n=32)$ featured an average response to omissions that was $0.782 \pm 0.031$ auROC (response to actual Suc delivery was $0.855 \pm 0.024 ; p<$ 0.01 , Wilcoxon signed-rank test). The population of neurons with inhibitory matching firing $(n=18)$ had an average response to omission of $0.312 \pm 0.042$ (response to actual Suc delivery was $0.251 \pm 0.025 ; p>0.05$, Wilcoxon signed-rank test). Responses to Suc and omissions were not necessarily identical $(42.0 \%, 21$ of 50 all matching neurons, had a significantly different response in the two conditions); however, a regression analysis (Fig. 10B) confirmed a significant degree of similarity (all neurons: $R^{2}=$ $0.373, p<0.01$, bootstrap; only excitatory response: $R^{2}=0.367$, $p<0.01$, bootstrap; inhibitory response: $R^{2}=0.269, p<0.05$, bootstrap). This behavior was specific for matching cueresponsive neurons, as analysis of population activity evoked by Suc and omissions in non-cue, Suc-responsive neurons revealed a dramatic difference between the two conditions (non-cueresponsive neurons excited by Suc: $n=32$; Suc, $0.715 \pm 0.018$; Omission, $0.553 \pm 0.020 ; p<0.01$, Wilcoxon signed-rank test; non-cue neurons inhibited by Suc, $n=18$; Suc, $0.403 \pm 0.006$,
Omission, $0.434 \pm 0.012 ; p<0.05$, Wilcoxon signed-rank test; Fig. 10A, right). The population response to omissions was $62.5 \%$ smaller than responses to Suc in non-cue-responsive neurons, compared with $21.6 \%$ in the matching neurons. Regression analysis between firing to Suc and to omitted Suc in non-cueresponsive neurons revealed a minimal correlation between the two conditions; $R^{2}=0.034$ ( $p<0.05$, bootstrap) when computed for all the non-cue-responsive neurons, $R^{2}=0.008(n=$ $48, p<0.05$, bootstrap) for non-cue-responsive neurons that were excited by Suc, and $1.16 \times 10^{-5}(n=44, p>0.05$, bootstrap) for non-cue-responsive neurons that were inhibited by Suc.

A frame-by-frame image analysis of orofacial movements was performed to determine whether the similarity in responses with omissions could be related to a similarity in mouth movements. Figure $10 \mathrm{C}$ shows mouth movements compared in the selfadministration (dark gray) and omission (light gray) trials. Contrary to neural responses of matching neurons, evoked mouth movements in the two conditions were dramatically different (particularly in the first 250-300 ms after lever pressing) with larger and rapidly emerging movements triggered by the actual self-administration of Suc and only slow and small movements triggered in omission trials (mouth movement size: Suc, $0.222 \pm$ 0.023 change in intensity $(\Delta \mathrm{I}) / \mathrm{pixel} /$ frame; Omission, $4.74 \times$ $10^{-4} \pm 0.014 \Delta \mathrm{I} /$ pixel/frame; $266 \mathrm{~ms}$ analyzed; $n=38 ; p<0.01$, Wilcoxon signed-rank test; mouth movement latencies: Suc, $279.0 \pm 22.5 \mathrm{~ms}$; Omission, $515.2 \pm 19.3 \mathrm{~ms} ; n=38 ; p<0.01$, Wilcoxon signed-rank test). Figure $10 D$ shows raster plots and PSTHs for two representative neurons, a Suc_cue-responsive neuron (left) and a non-cue-responsive neuron (right), in response to self-administration of Suc and omission trials. Visual inspection of the representative neurons clearly shows a response to omissions in the cue-responsive neurons and no response in the case of the non-cue-responsive neurons. Notably, the response to omission is not simply a continuation of the cue responses, but rather a significant elevation in firing rates relative to preomission time. 
A Population PSTHs for Sucrose Expected vs. Omission Responses

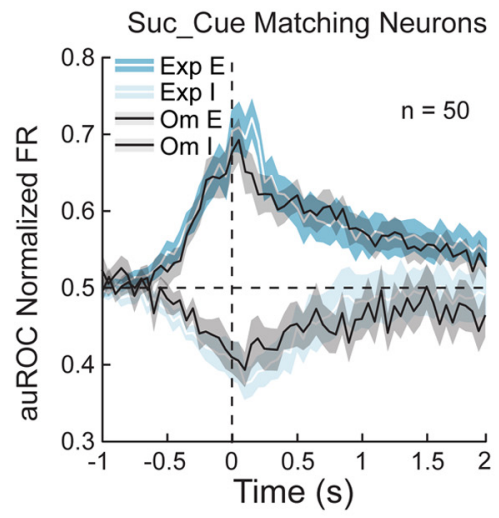

\section{C}

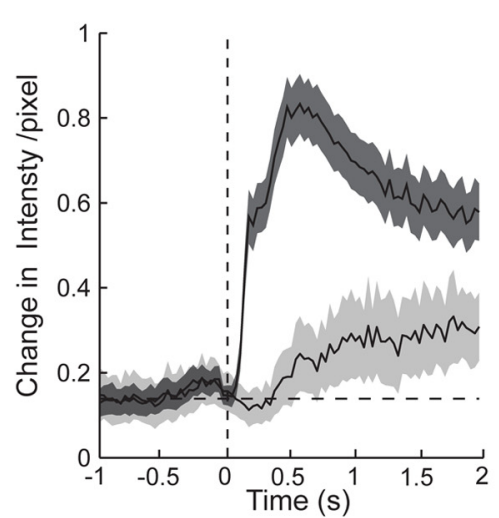

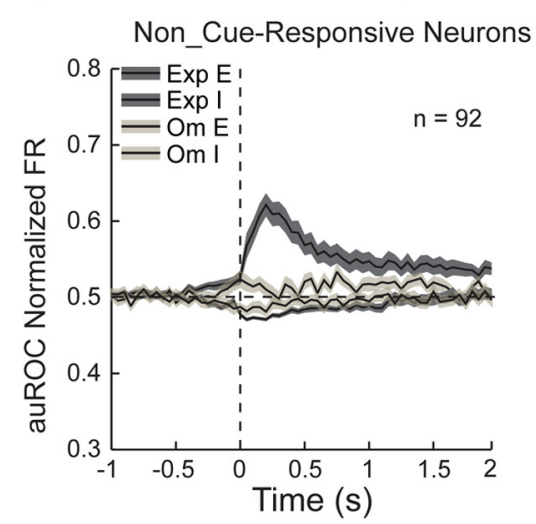

D Suc_Cue Matching Neuron

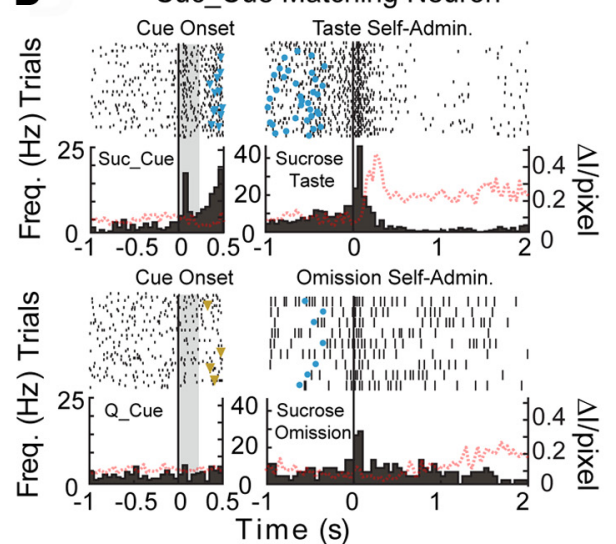

B Scatter of Suc Taste \& Omission for Sucrose Matching Neurons
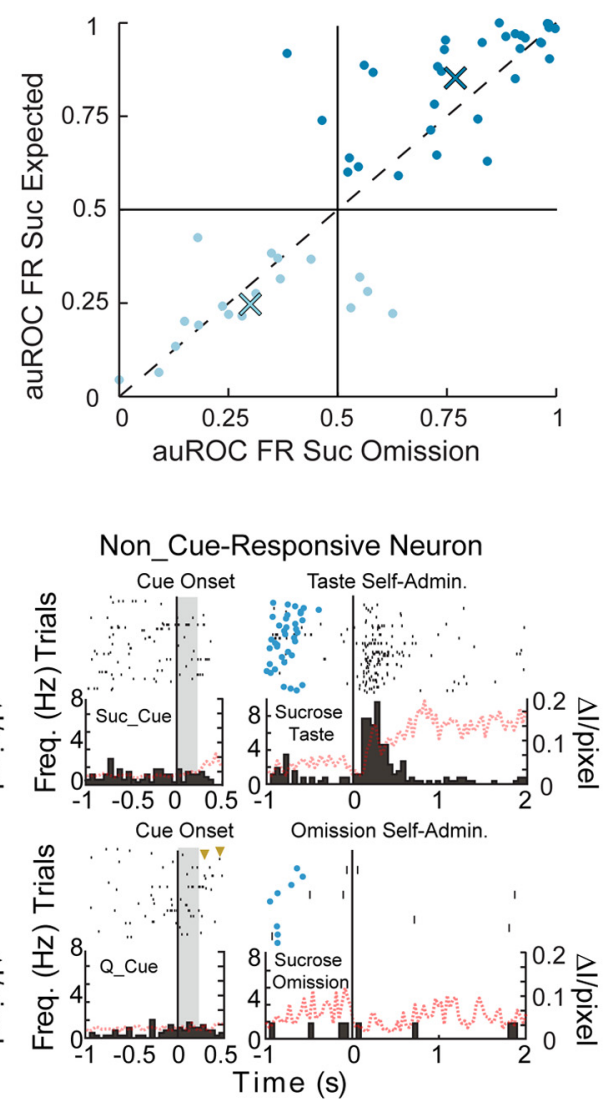

Figure 10. Matching neurons encode sucrose even in its absence. $\boldsymbol{A}$, Population PSTH in response to Suc self-administrations and omissions for matching neurons (left) and non-cue-responsive neurons (right). Cyan traces in left panel, Responses to self-administered Suc in matching neurons; light gray traces in left panel, responses to omissions in matching neurons; dark gray traces in right panel, responses to self-administered Suc in non-cue-responsive neurons; light gray traces in left panel, responses to omissions in non-cue-responsive neurons; Exp E, excitatory responses to expected Suc; Exp I, inhibitory responses to expected Suc; Om E, excitatory responses to omissions; $0 \mathrm{~m} \mathrm{I,} \mathrm{inhibitory} \mathrm{responses} \mathrm{to} \mathrm{omissions;} \mathrm{shading}$ around traces, SEM; $x$-axis, time (dashed line at 0 , lever press; i.e., Suc self-administration or omission); $y$-axis, auROC. $\boldsymbol{B}$, Scatter plot comparing responses to Suc and omission for matching neurons. Dark cyan, Neurons with excitatory responses; light cyan, neurons with inhibitory responses; $x$-axis, auROC; $y$-axis, auROC. $C$, Comparison of mouth movements evoked by Suc (dark gray) and by omissions (light gray). Shading around traces, SEM; $x$-axis, time ( 0 , timing of lever press); $y$-axis for mouth movements, difference of intensity for adjacent pixels. D, Representative examples of a matching neuron (left panels) and a non-cue-responsive neuron (right panels). Raster plots and PSTHs in response to cues, self-administered Suc, and omission. Red dashed traces superimposed on PSTHs represent the time course of mouth movements. $x$-axis, time (vertical line at time 0 for cue responses, cue onset; vertical line at time 0 for Suc and omissions, lever pressing time); $y$-axis for rasters, trials; $y$-axis for PSTHs, firing frequency; $y$-axis for mouth movements, difference of intensity for adjacent pixels; cyan triangle, time of Suc self-delivery; gold triangle, time of Q self-delivery; cyan circle, time of Suc_cue onset.

Finally, an additional set of analyses was performed to investigate whether responses to omissions extinguished. To this purpose, the responses of matching neurons to repeated omissions of Suc were analyzed in the extinction sessions. Rats pressed the lever an average of $18.5 \pm 1.1$ trials following the Suc_cue after the beginning of the extinction procedure. As these lever presses were not followed by Suc, they are equivalent to omission trials. To assess extinction of omission responses, activity in excited matching neurons ( $n=23$ of 41 neurons) was computed for the first five and the last five lever presses following the Suc_cue. This comparison showed a $41.1 \pm 23.3 \%$ decrease in omission responses (as measured in the first $250 \mathrm{~ms}$ following lever press) between early and late lever presses (magnitude of responses relative to baseline: in the first five trials, $0.258 \pm 0.046$; in the last five trials, $0.152 \pm 0.0600 ; p<$ 0.05 , Wilcoxon one-sided signed-rank test), showing that the response to omission does extinguish before reaching the behavioral criterion for partial extinction. To assess how this compares to the extinction of the cue, the $250 \mathrm{~ms}$ of cueevoked activity was analyzed in the same trials. Cue-evoked activity did not show a significant difference between the first five and last five lever press trials $(9.00 \pm 22.40 \%$ decrease; magnitude of responses relative to baseline: in the first five trials, $0.221 \pm 0.041$; in the last five trials, $0.201 \pm 0.045 ; p>$ 0.05 , Wilcoxon one-sided signed-rank test) before reaching the criterion for partial extinction. As expected on the basis of the results presented in Figure 6, there was a significant difference in cue responses between the first five lever press trials and the nonpress trials after the criterion for partial extinction was reached (first five trials, $0.221 \pm 0.041$; partial_ext, 0.130 0.037; $p<0.01$, one-sided Wilcoxon signed-rank test).

Altogether, these analyses show that a large subset of Suc_cueresponsive neurons (i.e., those with matching responses to Suc_cue and Suc) produces Suc-like responses even in the absence of the reward and that responses to omission can extinguish more rapidly than cue responses. 


\section{Discussion}

In previous reports, we showed that neurons in GC respond to a single cue anticipating the availability of multiple gustatory stimuli (Samuelsen et al., 2012, 2013). In nature, however, cues are not just general preparatory signals; they frequently predict a specific outcome. To investigate whether GC neurons can encode specific expectations, we recorded single-unit responses in rats performing multiple variants of an auditory go/no-go task and a classical conditioning paradigm. The results presented here demonstrate that GC neurons can selectively respond to auditory cues anticipating different outcomes. Responses to specific cues could be interpreted in different ways. According to one view, GC neurons would selectively respond to cues anticipating highly palatable (Suc) or highly aversive (Q) tastants. According to a second interpretation, cue responses would reflect the anticipation of the presence or the absence of a palatable reward. While cue responses in the go/no-go task could relate to either one of these models, the presence of cue-specific responses in classically conditioned rats provides evidence in favor of the first view. Regardless of the specific interpretation, analyses of different behavioral sessions revealed that cue-selective responses are acquired with learning and extinguish, hence tracking the anticipatory value of the cue.

We also found that responsiveness to cues predicted how neurons respond to Suc. Neurons selectively encoding the Suc-predicting cue responded to Suc more strongly than neurons that were selectively activated by the Q-predicting cue. The majority of neurons selectively responding to the Sucanticipating cue had matching responses to Suc. A neuron inhibited by the Suc-anticipating cue would likely be inhibited by Suc; likewise, a neuron excited by the cue would likely be excited by Suc.

Two sets of experiments were performed to investigate how this population of matching neurons responded to violations of expectation. The first set relied on unexpected, uncued deliveries of Suc. The experiments revealed that responses to expected and unexpected Suc were not very different. Matching neurons showed only an early and small enhancement of responses to expected Suc. In the second set of experiments, we investigated how matching neurons encoded the unexpected absence of Suc. Matching neurons responded to omission of sucrose with patterns of activity that could be reminiscent of sucrose responses. While the exact nature of these responses is at the moment only a matter of speculation, they could be related to the activation of Suc representations. Of course, other interpretations of this pattern of activity, for instance "surprise signals," could be possible. Regardless of the exact nature of responses to omissions, it is interesting to note that they were observed only in cue-responsive matching neurons, as the other neurons typically did not respond to the omitted reward.

\section{Responses to specific cues}

Approximately $20 \%$ of the neurons in GC respond to general anticipatory cues (Samuelsen et al., 2012). A similar percentage of cue-responsive neurons was observed in the task presented here, in which cues specifically predicted different outcomes. As in the case with general cue responses, specific responses are not the result of conditioned mouth movements.

Analysis of the distribution of cue responses revealed that in the go/no-go task the majority of neurons are cue selective and that most of these are activated by the Suc-predicting cue. Cue selectivity does not depend on the specific task used to condition the cues. Indeed, control experiments relying on classical condi- tioning showed that cue-specific neurons were present also in the absence of lever pressing. A smaller bias for the Suc-predicting cue was observed in this condition compared with the large bias observed in the go/no-go task. The large number of Suc_cueselective neurons in the go/no-go paradigm does not simply depend on the motor act of pressing the lever. Analysis of neural activity related to spontaneous and uncued lever presses showed almost no modulation in cue-responsive neurons. It is possible that the additional bias might be due not to the act of pressing itself, but to the cued-triggered intention to perform a consummatory act. According to this view, cue-related activity in GC may be important not only for its anticipatory value, but also for outcome-directed action. Whether these two functions are independent or integrated remains to be studied.

Regardless of the potential relationship between cue responses and executive functions, evidence of cue specificity in the classical conditioning paradigm clearly supports the link between cue responses and anticipation of sucrose and quinine administration.

\section{Learning and anticipation}

The appearance of cue responses is linked to learning. Previous experiments showed that the number of neurons responding to general cues increases dramatically once animals learn that the cue predicts the availability of tastants (Samuelsen et al., 2012). Here, we did not observe a significant increase in the overall number of cue-responsive neurons as animals learn the go/no-go task. This result is likely linked to pretraining on a single cue before training on the go/no-go task. On the basis of prior data (Samuelsen et al., 2012), it is reasonable to speculate that the recruitment of the pool of cue-responsive neurons had already occurred during the initial single-cue pretraining. The present data suggest that successive learning of the go/no-go task does not result in an additional expansion of the pool of cue-responsive neurons, but rather in a refinement of the cue selectivity within this pool. Indeed, here we demonstrate that the appearance of cue selectivity is largely dependent on learning. When recorded in a subset of first go/no-go training sessions (i.e., those in which the animals do not show any sign of learning), the majority of cue responses were found to be nonselective. These nonselective responses likely reflected the general expectation of a tasting solution learned during pretraining, with a single cue predicting both Suc and Q. We did not track the emergence of nonselective responses in this study; however, previous work established that general cue responses emerge with learning (Samuelsen et al., 2012) and that they can occur independently from lever pressing (Samuelsen et al., 2013). The proportion of neurons showing cue-selective responses increased significantly in the first sessions in which animals showed signs of learning. Analysis of the time course of initial sessions revealed a progressive increase in cue selectivity that paralleled learning. Within-session changes in cue responsiveness were also seen when the contingencies between cues and taste were partially extinguished. The amplitude of responses evoked by the Suc_cue significantly decreased once the performance dropped $<40 \%$. This reduction was not the result of time passing, as no change in the magnitude of cue responses was observed over time.

\section{Relationship between cue and taste responses}

Current models of sensory processing emphasize the importance of expectation in shaping the activity of sensory areas (Rao and Ballard, 1999; Engel et al., 2001; Zelano et al., 2011). Here we show that at the single-neuron level there is a relationship be- 
tween responsiveness to anticipatory cues and to taste. Our data demonstrate that the firing of a neuron in response to cues can predict its response to Suc. Neurons specifically activated by the Suc_cue respond more strongly to Suc than neurons activated by the Q_cue. Additionally, the sign of the response to the Sucanticipating cue predicts the sign of the response to Suc. Neurons excited by the Suc_cue are likely to respond to Suc with an increase in firing rates; similarly, neurons whose firing activity is depressed by the Suc_cue are more likely to be inhibited by Suc. The skewing toward excitatory or inhibitory responses was specific to cue-responsive neurons, as little bias toward excitation or inhibition was seen in neurons that are not cue responsive.

These anticipatory neurons were further investigated to determine how their evoked activity was influenced by expectation. Analysis of responses to uncued presentations of Suc revealed that the taste responsiveness of these neurons was only moderately influenced by expectation and that they responded slightly more to expected Suc administration than to unexpected Suc administration. This result indicates that these neurons are not encoding prediction error signals. Analysis of the entire population of neurons recorded in GC revealed a small group of neurons (9.2\%, 28 of 302 neurons) that responded exclusively to uncued, unexpected Suc. However, the vast majority of these neurons did not respond to anticipatory cues ( $92.8 \%, 26$ of 28 neurons). This result is consistent with prior proposals (Veldhuizen et al., 2011) suggesting the absence of classical prediction error neurons in GC, which is analogous to those seen in VTA (Schultz et al., 1997).

A second set of experiments investigated how matching neurons responded to the unexpected omission of the Suc reward. The responses of matching neurons to Suc omissions were reminiscent of their responses to Suc. Given this similarity, one could speculate that cues activated anticipatory representations that resulted in sucrose-like responses to omissions. These omission responses were observed only in matching neurons; neurons nonresponsive to cues showed very small changes in firing rates following omissions. A possible objection to omission responses in GC is that they could represent a response carried over from the cue. However, auditory cues were terminated at lever pressing; hence, no sound was present at the moment of the omission. In addition, the overall similarity in responses to expected and unexpected Suc in matching neurons clearly suggests that cue-evoked activity does not continue after taste delivery. Finally, visual inspection of omission-responsive neurons did not provide evidence for a continuation or slow decay of cue response into the omission period. Interestingly, responses to omissions appeared to be highly dynamic as they extinguished more rapidly than cue responses when Suc was consistently omitted.

\section{Conclusion}

Anticipatory cues provide information that allows animals to predict the availability and the identity of the substance to be ingested. Our results provide evidence for the presence of anticipatory signals in GC. These anticipatory signals could serve multiple functions, as follows: improve the accuracy and the speed of stimulus identification (Ashkenazi and Marks, 2004; Veldhuizen et al., 2011; Zelano et al., 2011); guide consummatory behaviors (Balleine and Dickinson, 2000; Parkes and Balleine, 2013); and influence the perceived hedonic value of fluids (Delamater et al., 1986; Nitschke et al., 2006; Small et al., 2008). The data presented here represent the basis for future investigations on the functional role of anticipatory activity in GC.

\section{References}

Ashkenazi A, Marks LE (2004) Effect of endogenous attention on detection of weak gustatory and olfactory flavors. Percept Psychophys 66:596-608. CrossRef Medline

Balleine BW, Dickinson A (2000) The effect of lesions of the insular cortex on instrumental conditioning: evidence for a role in incentive memory. J Neurosci 20:8954-8964. Medline

Chubykin AA, Roach EB, Bear MF, Shuler MG (2013) A cholinergic mechanism for reward timing within primary visual cortex. Neuron 77:723735. CrossRef Medline

Cohen JY, Haesler S, Vong L, Lowell BB, Uchida N (2012) Neuron-typespecific signals for reward and punishment in the ventral tegmental area. Nature 482:85-88. CrossRef Medline

Delamater AR, LoLordo VM, Berridge KC (1986) Control of fluid palatability by exteroceptive Pavlovian signals. J Exp Psychol Anim Behav Process 12:143-152. CrossRef Medline

Engel AK, Fries P, Singer W (2001) Dynamic predictions: oscillations and synchrony in top-down processing. Nat Rev Neurosci 2:704-716. CrossRef Medline

Fontanini A, Katz DB (2005) 7 to $12 \mathrm{~Hz}$ activity in rat gustatory cortex reflects disengagement from a fluid self-administration task. J Neurophysiol 93:2832-2840. CrossRef Medline

Fontanini A, Katz DB (2006) State-dependent modulation of time-varying gustatory responses. J Neurophysiol 96:3183-3193. CrossRef Medline

Gutierrez R, Simon SA, Nicolelis MA (2010) Licking-induced synchrony in the taste-reward circuit improves cue discrimination during learning. J Neurosci 30:287-303. CrossRef Medline

Jones LM, Fontanini A, Sadacca BF, Miller P, Katz DB (2007) Natural stimuli evoke dynamic sequences of states in sensory cortical ensembles. Proc Natl Acad Sci U S A 104:18772-18777. CrossRef Medline

Katz DB, Simon SA, Nicolelis MA (2001) Dynamic and multimodal responses of gustatory cortical neurons in awake rats. J Neurosci 21:44784489. Medline

Maffei A, Haley M, Fontanini A (2012) Neural processing of gustatory information in insular circuits. Curr Opin Neurobiol 22:709-716. CrossRef Medline

Moran A, Katz DB (2014) Sensory cortical population dynamics uniquely track behavior across learning and extinction. J Neurosci 34:1248-1257. CrossRef Medline

Narayanan NS, Laubach M (2009) Methods for studying functional interactions among neuronal populations. Methods Mol Biol 489:135-165. CrossRef Medline

Nitschke JB, Dixon GE, Sarinopoulos I, Short SJ, Cohen JD, Smith EE, Kosslyn SM, Rose RM, Davidson RJ (2006) Altering expectancy dampens neural response to aversive taste in primary taste cortex. Nat Neurosci 9:435-442. CrossRef Medline

Parkes SL, Balleine BW (2013) Incentive memory: evidence the basolateral amygdala encodes and the insular cortex retrieves outcome values to guide choice between goal-directed actions. J Neurosci 33:8753-8763. CrossRef Medline

Phillips MI, Norgren R (1970) A rapid method for permanent implantation of an intraoral fistula in rats. Behav Res Methods Instrument 2:124. CrossRef

Piette CE, Baez-Santiago MA, Reid EE, Katz DB, Moran A (2012) Inactivation of basolateral amygdala specifically eliminates palatability-related information in cortical sensory responses. J Neurosci 32:9981-9991. CrossRef Medline

Rao RP, Ballard DH (1999) Predictive coding in the visual cortex: a functional interpretation of some extra-classical receptive-field effects. Nat Neurosci 2:79-87. CrossRef Medline

Roesch MR, Calu DJ, Esber GR, Schoenbaum G (2010) All that glitters. Dissociating attention and outcome expectancy from prediction errors signals. J Neurophysiol 104:587-595. CrossRef Medline

Sadacca BF, Rothwax JT, Katz DB (2012) Sodium concentration coding gives way to evaluative coding in cortex and amygdala. J Neurosci 32: 9999-10011. CrossRef Medline

Saddoris MP, Holland PC, Gallagher M (2009) Associatively learned representations of taste outcomes activate taste-encoding neural ensembles in gustatory cortex. J Neurosci 29:15386-15396. CrossRef Medline 
Samuelsen CL, Gardner MP, Fontanini A (2012) Effects of cue-triggered expectation on cortical processing of taste. Neuron 74:410-422. CrossRef Medline

Samuelsen CL, Gardner MP, Fontanini A (2013) Thalamic contribution to cortical processing of taste and expectation. J Neurosci 33:1815-1827. CrossRef Medline

Schoenbaum G, Roesch M (2005) Orbitofrontal cortex, associative learning, and expectancies. Neuron 47:633-636. CrossRef Medline

Schultz W, Dayan P, Montague PR (1997) A neural substrate of prediction and reward. Science 275:1593-1599. CrossRef Medline

Small DM, Veldhuizen MG, Felsted J, Mak YE, McGlone F (2008) Separable substrates for anticipatory and consummatory food chemosensation. Neuron 57:786-797. CrossRef Medline

Stapleton JR, Lavine ML, Nicolelis MA, Simon SA (2007) Ensembles of gustatory cortical neurons anticipate and discriminate between tastants in a single lick. Front Neurosci 1:161-174. CrossRef Medline
Summerfield C, Egner T (2009) Expectation (and attention) in visual cognition. Trends Cogn Sci 13:403-409. CrossRef Medline

Travers JB, Norgren R (1986) Electromyographic analysis of the ingestion and rejection of sapid stimuli in the rat. Behav Neurosci 100:544-555. CrossRef Medline

Usher M, Cohen JD, Servan-Schreiber D, Rajkowski J, Aston-Jones G (1999) The role of locus coeruleus in the regulation of cognitive performance. Science 283:549-554. CrossRef Medline

Veldhuizen MG, Douglas D, Aschenbrenner K, Gitelman DR, Small DM (2011) The anterior insular cortex represents breaches of taste identity expectation. J Neurosci 31:14735-14744. CrossRef Medline

Yoshida T, Katz DB (2011) Control of prestimulus activity related to improved sensory coding within a discrimination task. J Neurosci 31:41014112. CrossRef Medline

Zelano C, Mohanty A, Gottfried JA (2011) Olfactory predictive codes and stimulus templates in piriform cortex. Neuron 72:178-187. CrossRef Medline 